\title{
Unobserved Heterogeneity and Reserve Prices in Auctions
}

James W. Roberts

Duke University

November 6, 2009

ERID Working Paper Number 80

This paper can be downloaded without charge from

The Social Science Research Network Electronic Paper Collection:

http://ssrn.com/abstract=1695647

\section{Economic Research Initiatives at Duke WORKING PAPERS SERIES

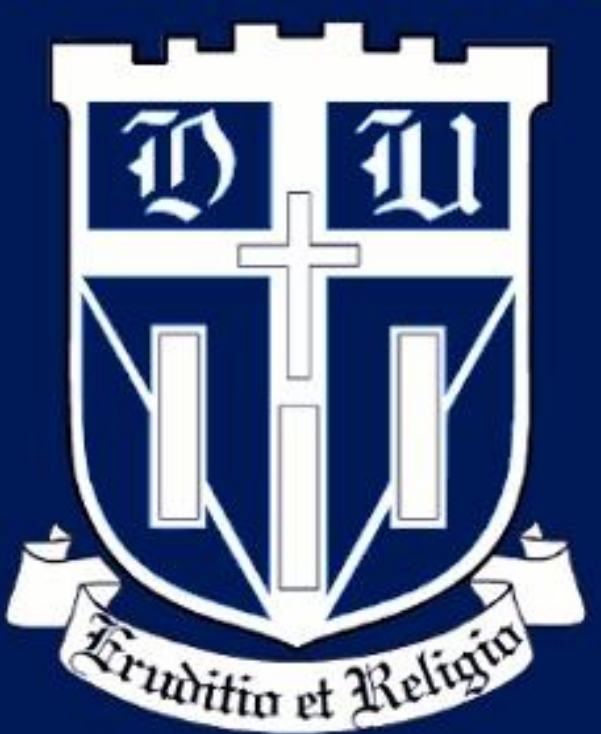




\title{
Unobserved Heterogeneity and Reserve Prices in Auctions
}

\author{
James W. Roberts*
}

November 6, 2009

\begin{abstract}
This study addresses the need to account for unobserved heterogeneity in auctions to improve our estimates of the distribution of bidder values. The method uses reserve prices to allow the distribution of bidders' private information to depend on the realization of the unobserved heterogeneity. The identifying assumption is that reserve prices are monotonic in the realization of unobserved heterogeneity and sellers are not required to set reserve prices optimally. The model can be estimated using only transaction prices. The paper proposes an estimation method and derives the asymptotic distribution of the proposed estimator. Working with data on used car auctions, the paper shows that controlling for unobserved heterogeneity affects estimates of the distribution of bidder values and impacts predicted outcomes dramatically.
\end{abstract}

JEL CODES: D44, L20, L62

Keywords: Auctions, Unobserved Heterogeneity

*Department of Economics, Duke University. Contact: j.roberts@duke.edu.

This paper has benefited from many helpful conversations with and insight from my dissertation committee: Robert Porter (chair), Igal Hendel, Ali Hortaçsu and Aviv Nevo. I also wish to acknowledge those generous with their time and advice including, but not limited to, John Asker, Matthew Gentzkow, Phil Haile, Ben Handel, Joel Horowitz, Tom Hubbard, Vadim Marmer, Rosa Matzkin, Ryan McDevitt, Kanishka Misra, Pablo Montagnes, Jason O’Connor, Mallesh Pai, William Rogerson, Aaron Sojourner, Kane Sweeney, Andrew Sweeting, Elie Tamer, Xun Tang and numerous seminar participants. Financial support from the Center for the Study of Industrial Organization is greatly appreciated. Finally, I am truly indebted to Joonsuk Lee for being so gracious in providing the data used in the paper. Any errors are my own. 


\section{Introduction}

Empirical auction work often estimates the underlying model primitives of bidder preferences to answer some counterfactual question. In this way the field is similar to many other areas of applied economics. However, as it has been recently noted (Einav and Nevo (2007) and Pakes (2008)), empirical auction research has not kept pace with other empirical branches of economics in its treatment of unobserved heterogeneity. In much of the empirical literature analyzing auctions economists make the implicit assumption that their information set about the items to be auctioned is the same as the auction participants' information set. This assumption is likely to be violated in many applications. Several papers address the problem, but they require rich data sets, potentially unpalatable modeling assumptions and ignore another potential source of information regarding the objects' unobserved characteristics: seller behavior. In this paper I loosen these restrictions and incorporate seller behavior in order to demonstrate how to obtain better, in the sense of consistency, estimates of demand in the presence of unobserved heterogeneity.

When the auction literature does control for unobserved heterogeneity, it only uses information from bidder behavior. The major insight that this paper brings is that we can also use information from seller behavior. If bidders observe and respond to a characteristic about the object that the econometrician cannot observe, it is likely that the seller, who as the object's owner is familiar with its intricacies, also observes this characteristic. While empirical auction work has moved forward by utilizing bidder behavior to grapple with unobserved heterogeneity, in this paper I show how to use the behavior of the seller, such as the realizations of one of his choice variables, to recover information about what the econometrician does not observe. One important choice variable of a seller is his reserve price (the price below which he will not sell the item), and in this paper I propose a general model of reserve price policy and bidding behavior that allows us to control for unobserved item heterogeneity when estimating the distribution of bidder values.

I present a general nonparametric model of reserve price-setting and bidding behavior, show that the model is identified and present Monte Carlo evidence that using reserve prices improves our estimation of the distribution of bidder values in the presence of unobserved attributes. To reduce dimensionality, I then introduce a single index that enters both the reserve pricing and bidding functions and I show that the distribution of bidder values is identified in this model. I then provide justifications for the single index assumption, one of which is that oftentimes the covariates entering the index are quality measures. Since I use this single index assumption in my estimation, I derive the asymptotic distribution of my estimator of the distribution of bidder values.

I then apply my method in an analysis of Korean used car auctions which present a textbook case for efforts to incorporate unobserved heterogeneity into our analysis. In these auctions owners of used cars employ the services of an auction house to sell their vehicles to used car dealers via a mechanism akin to an English button auction. I make sure to validate my modeling assumptions in the context explored. Among others, I attempt to justify the single index assumption, that both sellers and bidders observe and respond to what the economist does not observe, that conditional on an automobile's attributes bidders have independent private values and that bidders have unit 
demands. I also show why these used car auctions are an interesting and appropriate market in which to employ my methods. Aside from these auctions' importance to the overall automobile industry (the value of automobiles sold by auctions in the U.S. was almost $\$ 89$ billion in 2007 and used car dealers filled over $30 \%$ of their inventory via auctions), they are likely to be riddled with unobserved heterogeneity. That is, even after controlling for observable differences in cars, like make, model, age and mileage, there are likely to be differences in cars that are observable to auction participants, but are unobservable to the econometrician. I then demonstrate that my nonparametric estimates of the distribution of bidder values are dependent on the realization of unobserved heterogeneity.

After performing the estimation, I turn to utilizing my estimates to answer policy and counterfactual questions of interest and demonstrate the consequences of ignoring the presence of unobserved heterogeneity in these investigations. First, reserve prices are shown to be particularly useful when attempting to determine within-auction variation in bids when only single bids per auction are available in the data. On average, I show that utilizing reserve prices allows us to reduce our estimates of the within-auction variation in bids by an average of $75 \%$. Second, I show that the literature's previously held belief that a failure to control for unobserved heterogeneity leads to an overestimate of the variation in bidder values, due to attributing too much variation in bids to variation in values, does indeed hold in my estimation results. More generally, I show that when this is the case, we will tend to overestimate the amount of surplus accruing to the winning bidder. In my data, ignoring unobserved heterogeneity leads to an overestimate of bidder surplus of approximately $30 \%$. Third, I analyze the ramifications of ignoring unobserved heterogeneity when estimating the optimal reserve price. In my data this can lead to a substantial drop in expected revenues and an increased tendency for the item to go unsold. Fourth, I show that if the auction house faced no cost of price discriminating, it would be optimal for it to charge each seller a fixed fee and no commission. In reality, the auction house charges a $\$ 50$ fixed fee and collects $4.4 \%$ of the winning bid as commission. I claim that this is rational for the auction because of the costs it faces in deriving each car's optimal fixed fee based on its characteristics. The auction house faces these costs because of heterogeneity across automobiles, which it cannot observe without further inspection. I then seek to estimate the maximum potential gains from switching to the 0 -commission, individual fixed fee form of price discrimination from its current non-price-discriminatory pricing scheme. To do this, I parameterize seller demand for the auction house and use its current pricing scheme to uncover these parameters which can then be used to estimate the optimal fixed fees in the absence of inspection costs. I show that ignoring unobserved heterogeneity leads us to underestimate the potential gains from price discriminating which may be more than $5 \%$ of current revenues per car.

As auctions are more frequently used, economists will be increasingly called upon to analyze their outcomes and advise on their designs. In order to provide sound analysis and recommendations, it is essential to control for the presence of unobserved heterogeneity. This research illustrates how using reserve prices can assist in dealing with unobserved heterogeneity and shows how outcomes of interest depend on our acknowledgment and handling of it. 
The paper proceeds as follows: Section 2 places this paper in the context of the literature. Section 3 introduces the modeling framework used for estimation and addresses several questions of identification. Section 4 introduces the Korean automobile auction data. Section 5 discusses estimation, illustrates its results and brings these results to bear on several policy relevant questions. Section 6 concludes. Propositions and their proofs are presented in the appendix and all tables and figures appear at the end.

\section{Literature Review}

Accounting for differences in items being auctioned, which are unobservable to the econometrician, is an outstanding issue in empirical auction research and presents serious complications for empirical work. In this section I describe the existing literature on unobserved heterogeneity in auctions and highlight how this paper advances the current body of work. These contributions are generally a slackening of modeling restrictions and an easing of implementation through the use of seller behavior.

While economists have methods for dealing with observable heterogeneity between items, there is no universal approach for dealing with unobserved heterogeneity. This problem is compounded because auction participants generally have more information about the item for sale than the researcher. The often unstated assumption is that the economists' information set about the object coincides perfectly with that of the bidders, aside from their private signals, and therefore that any variation in bids across similar objects "on paper" derives solely from variation in bidder values. Recently there have been several attempts to deal with this issue. (Campo, Perrigne, and Vuong (2003), Bajari and Ye (2003), Hong and Shum (2002), Haile, Hong, and Shum (2006), Krasnokutskaya (2009) and Athey, Levin, and Seira (2004)). The first three papers use information provided by the number of bidders. The first in this group assumes that the number of bidders is a sufficient statistic for the unobserved heterogeneity while the second parametrizes the median of the bid distribution as being normally distributed with a mean dependent on the number of bidders in an auction.

In a very different approach to that presented here, Krasnokutskaya (2009) cleverly apples deconvolution techniques, the roots of which can be found in Li and Vuong (1998), and Li, Perrigne, and Vuong (2000) in the auction literature, to address unobserved heterogeneity. Like this paper, Krasnokutskaya's semiparametric method, which has recently been applied by other researchers, such as in Asker (2008), allows the unobserved heterogeneity to vary within auctions with the same number of bidders. However, there are several differences between our models. First, if she wishes to allow for asymmetric bidders, she requires data on multiple bids from the same auction to identify the distribution of bidder' signals, where as my method, though it requires data on reserve prices (or as will be discussed below, potentially other seller choice variables) only necessitates having data on the winning bid in an auction as well as the identity of the winner and second highest bidder. If symmetry is imposed her method can be adapted to the case with reserve prices as is done in 
Decarolis (2009). ${ }^{1}$ Second, her method assumes that a bidder's value (or cost since she is looking at procurement auctions, specifically highway maintenance and paving contracts) is the product of a bidder's private cost and a common unobserved component/cost. ${ }^{2}$ I, on the other hand, do not require specifying the functional relationship between private and common signals. Third, she requires bidders' signals to be distributed independently from the common component, where as I allow signals to depend on the unobserved common component.

Haile, Hong, and Shum (2006) present a similar idea for handling unobserved heterogeneity. Their paper is focused on developing a test for private vs. common values and in it they address the presence of unobserved heterogeneity in a similar way to this paper by modeling the number of bidders as being strictly increasing in the unobserved heterogeneity. They address the potential selection problem that participation may signal high unobservable realizations of the unobserved heterogeneity (which would happen if the value of the object needed to pass some threshold in order to be auctioned), something that I do not focus on in this paper. However, dealing with this selection problem may prove to be easier in this case since reserve prices are continuous, as opposed to the discreteness of the number of bidders. ${ }^{3}$ Moreover, they do not employ the single index model used below.

In another attempt to address the presence of unobserved auction level heterogeneity, Athey, Levin, and Seira (2004), in their study of timber auctions, assume a parametric form for the distribution of bids conditional on the unobserved heterogeneity. They then assume a distribution for this unobserved heterogeneity and form the unconditional likelihood based on their parametric assumptions. They also assume, which I do not, that the unobserved heterogeneity is orthogonal to the observable characteristics of the item being auctioned. The benefit of their specification of the distribution of bids conditional on unobserved heterogeneity and the distribution of unobserved heterogeneity is that when they recover the model's parameters using maximum likelihood, the unobserved heterogeneity term integrates out. Later, however, the authors still must incorporate the parameterized, and now estimated, distribution of unobserved heterogeneity when they run simulations. The authors readily admit that some of these assumptions are strong, in particular that the realization of unobserved heterogeneity is orthogonal to the observable item characteristics, but they defend such assumptions as being palatable "in light of most empirical work on auctions, which makes the even stronger assumption that there is no unobserved heterogeneity at all." Moreover, the authors provide substantial evidence that their parameterization is appropriate by comparing predicted and actual bid residuals. ${ }^{4}$

\footnotetext{
${ }^{1} \mathrm{~A}$ more flexible version of her model is presented in $\mathrm{Hu}$, McAdams, and Shum (2009) but they require data on three bids in an auction.

${ }^{2}$ As she notes, this multiplicative form assumption is justified if one believes that the cost distribution for the item is scaled by the common component, thus affecting the mean and variance of the cost distribution similarly. She also introduces a slightly less restrictive form in which the mean and variance of the cost distribution are affected by the common component in orthogonal ways by letting there be a two-dimensional common component: one affecting average cost and one affecting the individual cost deviations, though this requires the stronger assumption that both components of the common shock are distributed independently from one another as well as from the private signals.

${ }^{3}$ I thank Phil Haile for pointing this out.

${ }^{4}$ In another defense of the authors' empirical work, their goal is to calibrate the distribution of bidder values in what are likely to be competitive (sealed) auctions to then make out-of-sample predictions to assess the competitiveness of
} 
While it may be tempting to suggest a "fixed effects" approach to the problem, a common solution to the problem of unobserved heterogeneity in other applied fields, there are at least three concerns with this proposal. First, using a linear model to incorporate the fixed effects may be unattractive in certain applications. Second, if we suggest a nonlinear incorporation of the fixed effects then we introduce the familiar "incidental parameters" problem, originally addressed in Neyman and Scott (1948), which leads to bias in the parameter estimates. Third, often economists do not have the luxury of possessing multiple bids per auction, as is the case in the empirical application in this paper, yet we still wish to account for unobserved heterogeneity.

Evidenced by the aforementioned literature, the current attempts to deal with unobserved auction heterogeneity use only bidder behavior. One might wonder whether we can use the behavior of another auction participant, who also observes what the econometrician does not, to grapple with unobserved heterogeneity. This paper seeks to answer this question in the affirmative through the utilization of seller behavior. ${ }^{5}$ Although this proposal and its details will be explained below, I reference a figure from a seminal paper in the empirical auction literature as motivation: Figure 3 from Laffont, Ossard, and Vuong (1995), which can be found on p. 978 in their Appendix A. The paper looks at Dutch auctions of eggplants in France. The picture lists the auctions in chronological order and plots for each one the winning bid and reserve price which clearly move in tandem. While in a Dutch auction, unlike the English auction studied below, changes in the winning bid might be induced solely by exogenous variation in reserve price, if this were the case, one would also expect variation in the fraction of items sold, which according to the authors does not happen. Therefore, while the auctions may seem similar on paper, there is likely to be something that is different from auction to auction which is recognized and responded to by seller and bidder alike.

The similarity between existing work in structural industrial organization should be noted. Using reserve prices to "control" for unobserved heterogeneity in the bidding functions closely mirrors the conceptual framework of Olley and Pakes (1996) and was originally suggested to be of use in auctions by Athey and Haile (2006). Another related attempt to use this approach in the structural econometric auction literature is by Guerre, Perrigne, and Vuong (2009), which relies on monotonicity of the unobserved heterogeneity in the number of bidders. One advantage of using reserve prices over the number of bidders is that many times the number of potential bidders in an auction is unobservable.

It is important to note that while this section has highlighted the ways in which I attempt to advance the literature, the current techniques of dealing with unobserved heterogeneity clearly demonstrate the penalty of ignoring its presence in our models. The results of Krasnokutskaya (2009) and Asker (2008) elucidate the severity of "assuming the problem away" in their estimation

other potentially collusive (open) auctions.

${ }^{5}$ In my use of seller behavior, a paper which is more tangentially related is Lewis (2007)'s analysis of eBay Motors. In his model the seller has private information which is signaled to the bidders and the economist observes this signal. In this way Lewis considers a different signaling game altogether and while my model is more restricted than his since I do not allow the seller to have this private information, I provide support for this presumption in the empirical application. Another important difference is that the information bidders have about the automobiles varies in Lewis (2007), thereby justifying a common values model, whereas in the application below there is no reason to think that bidder's information sets vary. I provide further justification for the private values approach below. 
of mark-ups in government procurement auctions and the measurement of damages from bidding rings. 6

\section{The Model}

In this section I explain my strategy for using variation in the reserve price to account for unobserved heterogeneity in auctions. The model makes the reasonable assumption that while the econometrician may not be able to observe everything bidders do, it is likely that sellers have this information. Moreover, if this information is important to bidders, and thus affects how they bid, it is likely also to affect how the seller sets his reserve price. Therefore, I will use variation in the reserve price to help identify the unobserved heterogeneity across items. However, in so doing, I wish to avoid making stringent assumptions about seller behavior. For example, one major assumption that I wish to avoid making is that sellers set their reserve price optimally. This is because even if they knew how to do this, it is not clear what their objective function is in many contexts since the most frequently used optimal reserve pricing formula relies on the seller being a monopolist selling a single object, often an incorrect supposition. Therefore, I only assume that a seller's reserve price is monotonic in the quality of the item. Moreover, I will not assume anything about the separability or additivity of the unobserved component.

This section is divided into two parts. The first develops and shows identification of a more general model to control for unobserved heterogeneity. The second introduces a technique to reduce dimensionality and this is the model on which the empirical work is built.

\subsection{Identification of the General Model}

To introduce notation, let bidders be indexed by $i$ and auctions by $t=1, \ldots, T$. In the auctions considered here, one indivisible good is auctioned off to a bidder from a collection of $n \in\{\underline{n}, \ldots, \bar{n}\}$ bidders. Let the number of bidders in an auction $t$ be given by $N_{t}$, making the number of competitors faced by bidder $i$ in auction $t$ equal to $N_{t}-1$. In the empirical section I consider used car auctions. For each car there are some observed characteristics $Z_{t} \in \Re^{K}$ and an unobserved (to the econometrician) characteristic $U_{t} \in \Re .{ }^{7}$ Both the seller and the bidders observe both $Z_{t}$ and $U_{t}$, even though the econometrician only observes $Z_{t}$. Let $U$ be distributed according to the distribution function $F_{U}$. At each auction upon observing the cars' characteristics $Z$ and the realization of $U$, bidders receive independent and private signals $S$, which could be considered their type, which is

\footnotetext{
${ }^{6}$ Finally, although the strategy is not employed in the paper, the work of Khan (2001) can be adapted so one can include a detailed data set in the semi-nonparametric estimation of a nonlinear function's non-additive error term by using a linear index and the techniques of maximum rank correlation estimation to develop a different estimation technique. There are obvious restrictions, however, in mandating that items' covariates should enter a bidding or reserve price function through a linear index which may be especially unattractive if one doesn't believe that these covariates form an index of quality.

${ }^{7}$ The assumption that unobserved heterogeneity is single valued is a restriction that will be maintained throughout the empirical portion of the paper. However, below I show how in principle we could relax this assumption and still identify the model should the necessary data be available.
} 
distributed according to the function $F_{S} \cdot{ }^{8} \mathrm{I}$ am assuming, therefore, that bidders are symmetric in their value distribution. Each bidder has some value for the object $V_{i}$ and I assume that the random variables $\left(S_{1}, \ldots, S_{n}, V_{1}, \ldots, V_{n}\right)$ are affiliated, where this is loosely taken to mean that if one of these variables takes on a higher value, then there is a greater probability that other variables also assume a higher value. ${ }^{9}$ Moreover, signals are informative in that, conditional on any realizations of his competitors signals, the expectation of a bidder's value is assumed to be increasing in his signal.

For now, consider the case when bidders have independent private values (IPV) so that:

$$
E\left[V_{i} \mid S_{1}=S_{1}, \ldots, S_{n}=s_{n}\right]=E\left[V_{i} \mid S_{i}=s_{i}\right]=s_{i}
$$

and let the auction format be any one in which bidders submit bid $B$ according to some function $B=\beta(Z, U, S)$.

Now, suppose that sellers set reserve prices $R$ according to $R=m(Z, U)$ where I further assume:

Assumption S1 For all values $z$ of $Z, m(Z, U)$ is strictly increasing in $U$.

Therefore, I am assuming that the function $m(\cdot)$ does not vary across sellers. This is a restriction that could be relaxed should the data set contain the necessary information about the sellers or contemporaneous auctions to allow it.

It is important to note that I am not requiring sellers to set reserve prices optimally. I assume that, ceteris paribus, a higher realization of the unobserved heterogeneity leads the seller to set a higher reserve price. This is simply a restriction on the function $m(\cdot)$ which is intuitively appealing and particularly inoffensive when we think of the unobserved heterogeneity as some index of unobserved quality. ${ }^{10}$ However, as the following proposition shows, even when we do assume that sellers set their reserve price optimally, Assumption S1 holds for a large class of bidder value distributions. ${ }^{11}$ From the literature on optimal auctions (Bulow and Roberts (1989)) we the optimal

\footnotetext{
${ }^{8}$ Throughout the paper the dependence of this distribution on $Z$ and $U$ is suppressed for notational convenience.

${ }^{9}$ The formal definition of affiliation is given: random variables $W$ are affiliated if their joint distribution $f_{W}$ is such that for any $w_{1}$ and $w_{2}, f_{W}\left(w_{1} \vee w_{2}\right) f_{W}\left(w_{1} \wedge w_{2}\right) \geq f_{W}\left(w_{1}\right) f_{W}\left(w_{2}\right)$ where $\vee$ denotes the component-wise maximum and $\wedge$ denotes the component-wise minimum.

${ }^{10}$ One concern with this assumption is based on heterogenous costs of selling the cars elsewhere across sellers. The reserve prices set by the sellers reflect, at least in part, their outside value of retaining the car in the event that the car doesn't sell. If two sellers of similar cars have differing outside options, for example due to differences in their respective costs of selling the cars elsewhere, then it is possible that reserve prices are not increasing in $U$. This may happen if the owner of the car with a lower value of unobserved heterogeneity has a lower cost of selling the car elsewhere than does the seller of the car with the higher value of $U$. This possibility is ignored given the empirical application. One reason is that no information about individual sellers is available. I also justify the lack of a seller's private information impacting reserve prices below.

${ }^{11}$ Let $X \sim F_{X}$ and $Y \sim F_{Y}$. Then $X$ hazard rate dominates $Y$ if and only if $\frac{1-F_{X}(t)}{f_{X}(t)} \geq \frac{1-F_{Y}(t)}{f_{Y}(t)}$ for all $t$. It should be noted that a stronger requirement that distributions of values for higher realizations of $U$ maintain a hazard rate dominance over those for lower realizations of $U$, which is implied by affiliation, and immediately delivers the result in Proposition 1, is itself not very restrictive. First, it is satisfied for many of the distributions often used to parameterize bidder values when those distributions' parameters are specified as monotonic functions of $U$. This can be seen in one example from specifying the distribution of values to be Weibull with parameters that depend monotonically on $U$ such as $F(v \mid U)=1-e^{-\left[\frac{v}{\lambda(U)}\right]^{k}}$, for $v>0$, where $k>0, \lambda(\cdot)>0$, where $\lambda^{\prime}(\cdot)>0$. It can be shown that the mean of values when $U=u_{2}$ relative to the mean when $U=u_{1}$ is given by $\frac{\lambda\left(u_{2}\right)}{\lambda\left(u_{1}\right)}$ is greater than 1 when $u_{2}>u_{1}$, a sensible result. More to the point however, the inverse hazard ratio is given by $\frac{1}{k \lambda U^{-k} v^{k-1}}$ which
} 
reserve price, assuming that $F_{V}$ is strictly increasing and continuously differentiable, is given by:

$$
\begin{aligned}
\widehat{\rho} & =\operatorname{argsup}_{\rho \in[\underline{v}, \bar{v}]} \Pi\left(\rho ; \mathrm{v}_{0}, F_{V}\right) \\
& =\operatorname{argsup}_{\rho \in[\underline{v}, \bar{v}]}\left[\rho-\mathrm{v}_{0}\right]\left[1-F_{V}(\rho)\right]
\end{aligned}
$$

Intuitively, equation 2 says that the optimal reserve price is one that maximizes the profits of a monopolist facing a demand curve described by $1-F_{V}(\cdot)$ and with "marginal cost" given by $\mathrm{v}_{0}$. If $\mathrm{v}_{0}$ is the opportunity cost of not selling the car at the auction, we can think of $\mathrm{v}_{0}$ as the value to the seller of keeping the car if it failed to sell at auction. The optimal reserve price is given:

$$
r^{*}=\mathrm{v}_{0}+\frac{1-F\left(r^{*}\right)}{f\left(r^{*}\right)}
$$

Proposition 1. Let the cumulative distribution of bidder values $V$, conditional on the realization of unobserved heterogeneity, be $G \equiv F(\cdot \mid U)$, with associated density $g$, where the dependence on $U$ is noted, and let the seller have a valuation for the object equal to $v_{0}$. Denote $r^{*}\left(v_{0}, G, U\right)$ as the optimal reserve price set by such a seller facing bidders characterized by value distribution $G$ and a realization of unobserved heterogeneity $U$. If $V$ and $U$ are affiliated and $c(t, U)=t-\frac{1-G(t \mid U)}{g(t \mid U)}$ is increasing in $t$ for all $U$, then $\forall u_{1}<u_{2}, r^{*}\left(v_{0}, G, u_{1}\right)<r^{*}\left(v_{0}, G, u_{2}\right)$.

Note that the regularity condition on the function $c(t, U)$ is the familiar one introduced in Myerson (1981).

Despite the result of Proposition 1, I will continue to allow sellers not to set the optimal reserve price. Based on the results of the next proposition I will argue that if we make normalization assumptions on the functions $\beta(\cdot)$ and $m(\cdot)$ and some conditional independence assumptions regarding the distributions of $U$ and $S$, we can identify the distributions of both unobserved heterogeneity and bidder signals without assuming independence between $S$ and $U$. The results build on the work of Matzkin (2003). Suppose that we decompose observable characteristics into two sets of variables: $Z=\left(Z_{0}, Z_{1}\right)$.

Proposition 2. Let Assumption $S 1$ hold and assume $(i) \exists \overline{z_{1}}$ such that $\forall U$ and $\forall z_{0}, m\left(z_{0}, \overline{z_{1}}, u\right)=$ $u$ and $(i i) U$ is independent of $Z_{1}$ conditional on $Z_{0}$. Then $F_{S \mid(Z, U)}(\cdot)$ is identified from observations of the transaction price and the reserve price in first and second price auctions.

Therefore, using only data on reserve prices and winning bids, the distribution of bidders' private values, conditional on observable auction covariates and the realization of unobserved heterogeneity, is identified. ${ }^{12}$ The proof of identification also suggests a two step estimation procedure whereby the

is increasing in $U$, delivering hazard rate stochastic dominance. Second, many of the properties that seem sensible when incorporating unobserved heterogeneity are maintained under the assumption of hazard rate dominance. For example, if bidder values are denoted by the random variable $V^{1}$ when the unobserved heterogeneity is $u_{1}$ and $V^{2}$ when it is $u_{2}$ and $u_{1}<u_{2}$, so we assume that $V^{1}$ is hazard rate dominated by $V^{2}$, then (i) $\mathrm{E}\left[V^{1}\right]<\mathrm{E}\left[\mathrm{V}^{2}\right]$ and (ii) the average transaction prices will be greater, in 1st price, 2nd price, Dutch and English auctions when $U=u_{2}$.

${ }^{12}$ One should note that the normalization required in the proposition is one that is implicitly assumed in many functional forms. For example, if we let $Z=Z_{1}$, both $R=Z U$ and $R=\gamma Z+U$ satisfy the restriction when $\bar{z}=0$. 
econometrician first estimates the distribution of unobserved heterogeneity and the reserve price function $m(\cdot)$. This allows him to "back out" the realization of the random variable $U$ in each auction since with $m(\cdot)$ known, for any $R$ and $Z$, there is a unique solution to $R=m(Z, U)$. Then he can estimate the distribution of $S$ conditional on some auction covariates and the realization of $U$ using the distribution of winning bids for those auction covariates and the realization of $U$. To illustrate this procedure, I perform a Monte Carlo study in the appendix. ${ }^{13}$

\subsection{Dimension Reduction: a Common Single Index}

While the two step estimation procedure outlined above is quite general, it may be difficult to apply this nonparametric routine when there are many observable dimensions along which objects differ. Therefore, I wish to introduce a procedure which is similar in spirit but allows us to incorporate a high dimensional $Z$. Thus, in this section I simplify the estimation procedure by noting that we can combine the covariates of the item into an index and this index will affect both the reserve price set by the sellers and the bids placed by the bidders. In this section, I first seek to convey the idea by assuming that the reserve price and the bidding functions will only be affected by the single index. I then highlight the restrictions and flexibility of this modeling assumption. After this I enrich the model by allowing other covariates outside of the single index to affect reserve prices and bidding.

To begin, assume that sellers set their reserve price according to the following function:

$$
R=m(h(Z, U))
$$

where $m(\cdot)$ is strictly monotonic. Bidders bid according to:

$$
B=\beta(h(Z, U), S)
$$

where $\beta(\cdot)$ is monotonic and where, just to emphasize, $\beta(\cdot)$ and $m(\cdot)$ are entirely different functions. ${ }^{14}$

I will address the restrictions placed on seller and bidder behavior in the context of the empirical portion of the paper: used car auctions (the data for which will be introduced in Section 4). The first assumption to note is that the same aggregator $h(Z, U)$ appears in both functions. This places very little restriction on the behavior of bidders and sellers as the following thought experiment will

\footnotetext{
${ }^{13}$ One extension of the model is to consider settings with multi-dimensional unobserved heterogeneity. It seems as though the previous identification result can be generalized to this setting, but this is only speculative. Suppose, for example, that the signal $U \in \Re^{L^{1}}, L^{1}>1$. What one could require is that there exist as many policy variables (denoted by what is now a vector $R$ ) as there are dimensions of $U, L^{1}$, and that for each component of $R \in \Re^{L^{2}}, L^{2} \geq L^{1}$, say $R_{l}$, there exist functions $R_{l}=m_{l}\left(Z, U_{l}\right)$ which are monotonic in $U_{l}$. Then if we allow the bids to depend on the entire vector $U$, we can simply perform the "first part" of the above proof $L^{1}$ times to each of the functions $m(\cdot)$ in order to uncover all of the realizations of unobserved heterogeneity. Still, this remains to be shown. One setting where we might want to consider such a model is eBay, where sellers can choose not only reserve prices, but also auction length, whether or not to display pictures of the item, whether or not to have the object insured, shipping charges, etc.

${ }^{14}$ Without loss of generality, since both $m(\cdot)$ and $\beta(\cdot)$ are monotonic, we could assume that $R=h(Z, U)$. However, in order to be explicit and maintain notational consistency, I will not relax this assumption now.
} 
reveal. Consider two cars with characteristics $\left(Z_{1}, U_{1}\right)$ and $\left(Z_{2}, U_{2}\right)$ such that:

$$
Z_{1}>Z_{2} ; U_{2}>U_{1} ; h\left(Z_{1}, U_{1}\right)=h\left(Z_{2}, U_{2}\right)
$$

Then it must be the case that $R_{1}=R_{2}$ and that for a bidder with $S, B_{1}=B_{2}$. Moreover, any movement within the index must move both the bids and the reserve prices, though certainly not by the same amount. ${ }^{15}$

Another assumption is that bidder and seller characteristics do not enter either the reserve pricing or bidding functions. To the extent that there may be strategic interactions among bidders based on repeated interaction or varying levels of competition in their home markets, one might think that these covariates should enter the bidding function. However, since the data come from an English auction where bidders bid their values truthfully, I will not need to consider the way that bidders incorporate information about the distribution of bidder specific covariates into the model. Moreover, since bidder covariates don't affect bidding behavior, the sellers' reserve pricing should also remain unaffected by these characteristics. We can further support the claim that bidders don't take into account the observable differences in their competitors since, as will be explained more fully below, they cannot discern which of their potential competitors is currently bidding against them. The assumption that a seller's covariates don't enter the reserve pricing function is also a restriction. As was mentioned above, heterogenous costs of selling the car elsewhere across the seller population may violate the underlying assumption that reserve prices are increasing in $U$. Unfortunately, the data needed to control for such differences is not available in the application below, but should it be in other contexts then these heterogeneous costs could, in principle, be controlled for. I do justify that there is no private signal impacting reserve prices in Section 4.2. However, despite these explanations for excluding bidder and seller covariates from impacting the reserve pricing and bidding equations, the model still could incorporate characteristics of the seller or bidders that are observable to each auction participant. It can do so through the unobserved heterogeneity, if we believe that this information can be compiled through such a uni-dimensional statistic.

Finally, the assumption that both $m(\cdot)$ and $\beta(\cdot)$ incorporate $Z$ and $U$ through $h(\cdot)$ is a restriction. For instance, we might want to let $B=\beta(Z, U, S)$. This is less restrictive, but since $Z$ and $U$ are likely to be quality measures, compiling them through $h(\cdot)$ seems fairly innocuous. Another complaint about the function $h(\cdot)$ might be that it should vary across types of sellers and bidders if there is horizontal differentiation in automobile dealerships. This is plausible since even though much of the data are quality measures, which would vertically differentiate cars, there might be a concern that certain bidders, who are automobile dealers, specialize in certain types of cars, and therefore some kind of random coefficients model would be more appropriate. Unfortunately, I cannot identify different types of bidders in my data, so for the time being I will proceed with

\footnotetext{
${ }^{15}$ Finally, if we require $R$ to be optimal for the seller, although not requiring it to be the optimal reserve price defined in Equation 3, then it cannot be the case that $h(\cdot)$ is not the same function for the seller and the bidders. Consider $R_{1}$ set in response to $\left(Z_{1}, U_{1}\right)$. If a new car with characteristics $\left(Z_{2}, U_{2}\right)$ alters bidding behavior but not seller behavior since they may have different $h(\cdot)$ functions, then $R_{1}$ could not have been optimal in the first place.
} 
keeping $h(\cdot) .{ }^{16}$

With the assumptions addressed, I now highlight the flexibility of this specification. Focusing on the sellers, I do not assume they set reserve prices optimally. I only assume that they are monotonic in the quality of the item. I also do not specify the form of competition by the sellers, though I refrain from allowing other items' characteristics to enter the reserve pricing function, except when "market conditions" are captured by $U$. I simply assume that the sellers face buyers whose values are increasing in the quality of the item for sale. For bidders, I have left the bidding behavior, and even the auction format itself, unspecified. Finally, I have said nothing so far that restricts correlation between any of the signals or between any signal and the covariates.

The payoff of this general specification is that should our ultimate goal be to recover an estimate of $F_{S}$, as is often the case in empirical auction work, the monotonicity of $m(\cdot)$ permits the following:

$$
h(Z, U)=m^{-1}(R)
$$

Then, by inserting equation 6 into equation 5 we can rewrite the bidder equation as:

$$
B=\beta\left(m^{-1}(R), S\right)
$$

Thus, we have eliminated the unobserved heterogeneity from the bidder function and now we can estimate the distribution of bidder values, $F_{S}$.

However, suppose we now wish to modify our analysis by allowing:

$$
\begin{gathered}
R=m(h(Z, U), \tilde{Z}) \\
B=\beta(h(Z, U), \tilde{Z}, S)
\end{gathered}
$$

where $\tilde{Z} \in R^{L}, L<K$ is potentially some subvector of $Z$. In words, we are allowing something beyond the function $h(\cdot)$ and a bidder's signal to determine his bid, and therefore the reserve price selected by the seller. Let $\Omega=\Re \times \Re^{L} \mathrm{x} \Re$. Then, for all $(h, \tilde{Z}, R) \in \Omega$ the monotonicity of $m(\cdot)$ implies that there exists a unique, continuously differentiable function $\varphi: \Re^{L} \mathrm{X} \Re \rightarrow \Re$ such that $\varphi(R, \tilde{Z})=h(Z, U)$ and

$$
B=\beta(\varphi(R, \tilde{Z}), \tilde{Z}, S)
$$

Again, the unobserved heterogeneity is "purged" from the bidding function. ${ }^{17}$

\footnotetext{
${ }^{16}$ Another implication of the model is that the marginal rate of substitution between $Z$ and $U$ given $S$ is independent of $S$ since: $\frac{\frac{\partial B}{\partial Z_{k}}}{\frac{\partial B}{\partial U}}=\frac{\frac{\partial B}{\partial h} \frac{\partial h}{\partial Z_{k}}}{\frac{\partial B}{\partial h} \frac{\partial h}{\partial U}}=\frac{\frac{\partial h}{\partial Z_{k}}}{\frac{\partial h}{\partial U}}$ and $h(\cdot)$ is not a function of $S$.

${ }^{17}$ Following the idea in footnote 13 , the process might be able to be generalized to include multidimensional unobserved heterogeneity. What I require is that there are $L^{1}$ signals which are distributed jointly independent, there exist enough seller choice variables $\left(>L^{1}\right)$ which are observable and that the following holds:

$$
\begin{gathered}
R_{l}=m_{l}\left(h_{l}\left(Z_{l}, U_{l}\right), \tilde{Z}\right), i=1, \ldots, L^{1} \\
B=\beta\left(h_{1}\left(Z_{1}, U_{1}\right), h_{2}\left(Z_{2}, U_{2}\right), \ldots, h_{L^{1}}\left(Z_{L^{1}}, U_{L^{1}}\right), \tilde{Z}, S\right)
\end{gathered}
$$

because I can apply the above inversion $L^{1}$ times, each time generating a new $\varphi_{l}(\cdot)$, each of which can be used to
} 
Before moving onto estimation, I now describe the data.

\section{Data Description}

In this section I introduce the data, show how it supports the contention that both bidders and sellers observe what the econometrician does not and justify the modeling assumptions made above in the empirical context.

The data in this paper come from used car auctions in South Korea from late 2001 through 2002. Auctions have been utilized to sell cars for at least 60 years and their use continues to grow rapidly. Automobile auctions are worth examining due to their size and importance to the overall automobile market, their relevance to the study of unobserved heterogeneity and their relationship to a host of interesting policy questions.

The National Automobile Auction Association (according to the NAAA 2007 survey) estimates that over 9.5 million cars, valued at nearly $\$ 89$ billion, were sold by auctions in the U.S. in 2007, a $2.2 \%$ increase over 2006 and a $5 \%$ increase over 2005. In wholesale used car sales, where dealers purchase inventory to fill their lots, auctions are still frequently used. The National Association of Automobile Dealers estimates that new and used car dealers acquired $32 \%$ of their used car inventory via auctions, up from less than 6\% in 1982 (see Taylor (2007) and Kontos (2006) for more details).

Aside from automobile auctions' size and scope, the analysis of such auctions presents a potential textbook case for investigating the problem of unobserved heterogeneity to the analyst and/or econometrician. It is likely that buyers and sellers present at the auction are able to observe aspects of the cars which affect their values and thus their bids and reserve prices, yet cannot be observed in even a rather extensive data set provided to the analyst. Examples of such unobserved qualities could be such things as rust, dents or tire quality, just to name a few. Therefore, it is likely that should the econometrician fail to account for such unobserved heterogeneity, his results from a host counterfactual experiments would be incorrect. ${ }^{18}$

\subsection{Summary of Data}

In this subsection I introduce the data and present some summary statistics. I then use the data to support the hypothesis that bidders and sellers observe and respond to unobservable characteristics of each car.

The used car auctions took place on a weekly basis at a particular auction house in Suwon, South Korea which is approximately an hour outside of Seoul by car, between October 10th, 2001 and November 27th, 2002. At this wholesale used car auction, both dealers and individuals may sell

substitute into equation 12 for $h_{l}(\cdot)$. This is only speculation and I leave a full proof of the idea, which is beyond the scope of this paper, to future work.

${ }^{18}$ Though outside the scope of this paper, we might be particularly concerned with the South Korean automobile market since on June 30th, 2007 the U.S. and South Korea signed a free trade agreement whereby South Korea agreed to remove its $8 \%$ tariff on U.S. made passenger vehicles and its $10 \%$ tariff on pickup trucks which is meaningful to U.S. producers who currently claim only a miniscule portion of the Korean auto market. 
cars but only dealers registered with the auction house may bid. During the 57 weeks covered, there were over 380 members registered with the auction house and this group was stable over the time period. Unlike the U.S., where it is common for dealers to sell both new and used cars, the dealers in this data set specialize in used cars only and according to sources these dealers are perceived to be sellers of high quality used cars. ${ }^{19}$ Also, car dealers in South Korea tend to be much smaller than their counterparts in the U.S. The auction house was set up by an affiliate of Daewoo and its main competitor was a similar, but much smaller, auction house (set up by Hyundai) an hour away. The auction house charges the seller $\$ 50$ for any car it auctions regardless of whether it sells and, in the event of sale, collects $2.2 \%$ of the transaction price from both the buyer and seller. The individual seller of the car has full control over the reserve price he sets, though in practice the auction house almost always provides guidance on this matter. Registered members receive information about cars that are going to be auctioned three days ahead of time. The information contains a description of the cars including make, model, mileage, age, etc. The cars have reserve prices, but these are not revealed to the buyers. If a dealer wishes to place a bid, he must send an agent to the auction house to be present at the time of the auction. ${ }^{20}$

On auction day, bidders show up three or more hours before the auction to inspect all of the automobiles being auctioned that day. When the auction begins, all bidders sit at tables in front of the car being auctioned. A screen at the front of the room displays the current bid for the car. The auction format used is a variant on the classic English button auction originally described in Cassady (1967) and analyzed in Milgrom and Weber (1982). ${ }^{21}$ The price of the item being auctioned rises continuously and in order to remain in the auction a bidder must keep his finger on a button beneath his table in front of him. Reentry of bidders is allowed which simplifies the strategies of the bidders since they do not need to condition on which bidders are currently "in" or "out" of the auction. The price for the car being auctioned rises in $\$ 30$ increments every three seconds provided that at least two bidders have their buttons depressed. Bidders cannot observe when their competitors have dropped out of the auction. ${ }^{22}$ A bidder wins if he is the last bidder with his finger on the button and he pays the current bid at this moment. The winning bidder is responsible for

\footnotetext{
${ }^{19}$ This is in contrast to other automobile markets sometimes considered. See for example Adams, Einav, and Levin (2007).

${ }^{20}$ An interesting practice by the auction house is that it permits a dealer to send multiple agents, each of whom can place separate bids on a particular car. This allowance has an ambiguous affect on transaction prices since to the extent that these cars have common value elements in them, a dealer which follows this strategy may hurt itself because the increase in perceived competition among other dealers due to this dealer sending multiple bidders might lead to an increase in the transaction price. On the other hand, if bidders become more afraid of the winners curse, they may bid less aggressively and thus lower the transaction price. Pinkse and Tan (2005) also provide a model where the winning bid does not necessarily increase in the number of bidders for reasons separate from the winner's curse, though they study first price auctions.

${ }^{21}$ The model of bidding behavior relies heavily on the auction operating as does the classic button auction. In the majority of empirical work with English auctions, researchers assume that the auction model really does follow the button procedure, even when this is far from the truth. For example, the auction may actually function as an open outcry auction. A benefit of the data in this paper is that it is one of the very few examples we have where the data generating process is literally a button auction. However, were it not the case, preventing us from relying on the equilibrium mapping between bids and unobserved demand, we could employ the now well known methodology of Haile and Tamer (2003).

${ }^{22}$ See Kim and Lee (2006) for additional descriptions of the auction house.
} 
transporting the automobile offsite.

During the period of study there were 28,334 passenger cars auctioned. The types of car auctioned were sedans, three and five door hatchbacks, jeeps, small minivans, minibuses, small trucks and motorbikes. ${ }^{23}$ In an effort to maintain a homogenous data set, the sample I analyze consists of over 8,500 sedans made by Hyundai, Daewoo (which was acquired by GM in 2001) and Kia (which was acquired by Hyundai in 1998) that had been previously owned for "regular" individual purposes, as opposed to being part of government or rental company fleets. Some summary statistics for the cars appear in Table 1. Importantly, in terms of bids, I only observe the transaction price (also referred to in the paper as the winning bid) and the identity of the winner and the second highest bidder. All variables are measured at the time of auction. Here AGE is the number of months since the car was originally manufactured, MILEAGE is the reading on the odometer in 100,000s of kilometers, AUTOMATIC is a dummy variable coding for whether the car has an automatic transmission $(=1)$ and PASSENGERS is a dummy variable coding for whether the car can seat five $(=1)$ or four passengers. I also have data on the model and colors of the car, though those statistics are not summarized in the table. The average car is a little under 6.5 years old, with around 100,000 kms (approximately 62,000 miles). Approximately two thirds of the cars have an automatic transmission and almost all hold five passengers. Cars tend to sell for a little under $\$ 3,000$. On average more than five bidders place bids on a car, and (not shown) over four place bids once the price has passed the reserve price. A person is counted as a bidder if they ever have their button depressed once the price has begun to rise.

Table 2 shows characteristics of the bidders. There are approximately 145 cars of the type I consider sold at each auction. There are 385 different bidders in the data and conditional on showing up, on average they win approximately 2 auctions per day and around 20 auctions of this type of car during the entire time I consider. There are some buyers who win much more than this, including 10 that win over 100 cars. In an effort to measure the overall bidding activity of the members, not just their winnings, I can (extremely) conservatively estimate how many bids they place by looking at all of the times the bidder is one of the two top bidders since this is all that I observe in the data. This measure shows that bidders are active during at least $17 \%$ of the days on average, though 12 are active on at least $90 \%$ of the days. Finally, I also have data on an imprecise measure of each bidder's location. I know in which of the 13 South Korean provinces each firm is located, and therefore, I can measure the distance from the centroid of each province to the auction house.

Figure 1 plots the winning bid and reserve price for similar cars (five passenger Hyundais with automatic transmissions) on a randomly chosen day, January 30th, 2002. As can be seen, there is evidence that sellers and bidders are observing the same information, which is unobserved by the econometrician, and responding to it in ways which are observable in the data.

To get a better feel for the factors affecting bids and the relationship between winning bids and reserve prices, tentatively explored in Figure 1, I begin with some basic analysis of price determinants

\footnotetext{
${ }^{23}$ Unlike the U.S., SUVs were not prevalent in South Korea at this time.
} 
for the automobiles. The results appear in Table 3. The estimated impacts of the cars' attributes are robust to different specifications. The price tends to fall by $3 \%$ for each additional month of its life. Its mileage also has a large impact on value, though not quite as pronounced since for each $100,000 \mathrm{kms}(62,000$ miles $)$ drops the price by around $3 \%$. There is a sharp difference in value between automatic and standard transmissions, with automatics commanding an almost $40 \%$ premium even after controlling for a car's make and model. Though it is not as meaningful since almost all cars hold the same number of passengers, cars that only hold four passengers sell for substantially less than those that hold five. Finally, and quite surprisingly, the distance of the winning bidder's headquarters from the auction site has a positive, but only marginally significant, and even less in the "economic" sense of the word, effect on his bid (and this does not seem to vary by adding higher powers of distance).

The last column of Table 3 includes the log of the reserve price as a covariate in an attempt to assess the comovement of it and the winning bid controlling for all of the other likely determinants of price. The winning bid and reserve price are highly correlated. In addition, there are other interesting things to note. First, all of the previously explored variables remain significant, though the magnitude of many of their impacts are lowered suggesting that the reserve price is not just some mechanical function of a car's characteristics. Second, the overall fit of the model jumps from, $85 \%$, already a high number, with the covariates now explaining almost $97 \%$ of the variation in price. All of the evidence from the last column suggests that the reserve price has great ability to control for unobserved variation in an item's "quality".

Table 4 explores the determinants of reserve prices. The results provide evidence that the factors affecting bidder values also affect reserve prices in a similar direction and by a comparable magnitude. This suggests that bidder and seller behavior in response to the observable qualities of the car are similar and thus lends support to the hypothesis that they may be similarly aligned over what is not observed by the econometrician.

Finally, to get a sense that the results in the first plot in Figure 1 weren't driven by differences in the mileage and age of cars, I also plot the residuals from regressions of the log of the winning bid and log of the reserve price on the observable characteristics of the cars. The results for the randomly chosen day used above are plotted in in the top right panel of Figure 1. The comovement evident in the plot is highly suggestive of the overlapping information of the seller and the bidder to which the economist is not privy. Also, not reported elsewhere, the correlation of these residuals is 0.9002 compared to a correlation of 0.9815 of the raw data.

The empirical work in the paper relies on auctions for which I have the winning bid conditional on the reserve price being met. From this I will rely on comovement between the winning bid and the reserve price to help control for the unobserved heterogeneity. However, we would want to see if this is the case for auctions that fail to meet the reserve price. For these auctions, it should also be the case that higher reserve prices tend to be associated with higher maximum, though ultimately failing, bids since there is still likely to be some unobserved characteristic that affecting bidders' bids and sellers' reserve prices. To check this I was able to obtain data on the highest bid placed 
for an object that did not sell (because the reserve price was not met) for a smaller time period: October 10th, 2001 to December 27th, 2001. During this time, 243 of the 2,142 cars auctioned, or $11.3 \%$, failed to sell. ${ }^{24}$ The bottom row of Figure 1 provides evidence that sellers and bidders are observing and responding to similar movement in what the econometrician does not observe. The bottom left figure is a plot of the reserve price and highest bid, in logs, for all auctions of Hyundai's with an automatic transmission during this time period. It is evident that the highest bid and reserve price are strongly correlated, just as these prices moved together in the top row of Figure 1.

For external validity, we also might wonder if there is something different about the cars that do not sell. To test this, I estimated a logit model of whether the car failed to sell as a function of the cars' characteristics and also the time of year. The results appear in Table ?? and will be referred to again in Section 4.2. The only characteristic that seems to matter is that older cars are more likely to fail to sell.

We can also see that the auctions that fail respond in a fairly similar manner to auction covariates as did the items that sold. This is borne out in Table 5 for both (logs of) highest bid and average bid. Though many of the impacts' significance are dampened, there is still a great deal of correlation between these bid statistics and the reserve price, as was the case in the regressions conditioning on a successful sale. Also, in the spirit of the top right panel in Figure 1, I plot the residuals from regressions of the highest bids and reserve prices on car characteristics for cars that failed to sell in the last plot in Figure 1. The correlation in these residuals is 0.9752 compared with a correlation of 0.9970 of the raw data. The results continue to support the similar possession of, and response to, information by bidders and sellers which is not available to the econometrician. ${ }^{25}$

\subsection{Does the Model Fit the Data?}

Before moving on to the estimation, it remains to be shown that the data fit the model and its assumptions described so far. In order to validate the model, I will address whether I can support the assumptions that (i) conditional on $Z$ and $U$ that the bidders at the used car auctions have independent private values, (ii) the results shown in Figure 1 aren't a mechanical result, (iii) the unobserved heterogeneity affects both the reserve price and the bids, (iv) we can utilize the single index model (v) bidders have a unit demand and (vi) sellers use the same reserve price setting function $m(\cdot)$. While there may be other concerns about the model, the verification of these assumptions should greatly strengthen the confidence in the estimation results below.

There are two justifications for assuming independent private values (IPV) among the bidders conditional on a car's characteristics. The first justification notes that while each bidder (all of whom are dealers) is interested in reselling the car should they win, each dealer has its own inventory, its

\footnotetext{
${ }^{24}$ The reserve price was binding in approximately $7 \%$ of these auctions. I am able to incorporate these auctions by using the second highest bid despite the fact it was less than the reserve price.

${ }^{25}$ Since the model relies on all bidders observing what the econometrician does not, we can also see if there is comovement between the second highest bid and the reserve price. Using the same diagnostic as above by regressing the reserve price and the second highest bid on the observables, we find a correlation in the observables of 0.8195 compared with a correlation of 0.98 in the raw data. This lends support to the underlying assumption of all bidders and sellers observing what the econometrician does not.
} 
own transportation cost and may cater to idiosyncratic local demands, ${ }^{26}$ thereby ruling out a pure common value component of the cars. The second justification is that in these auctions there is no reason to think that one bidder has a different information set about the car being auctioned than any other bidder. ${ }^{27}$ As is well known, the assumption of IPV does not rule out correlation in bidders' information or their values being commonly affected by characteristics of the item, provided that bidders do not have private information about the characteristics. So, the assumption that bidders all observe what the econometrician does not, and that this component affects their valuations, does not violate the assumption of IPV since bidders do not have private (from the other bidders) information about what the econometrician does not observe, in the same way they do not have private information about observed auction heterogeneity. This is in contrast to a market like eBay motors where buyers differ on many dimensions which are correlated with their information sets about the auctioned item. For example, Lewis (2007) notes that a common value model is more appropriate for eBay motors because bidders who live in close proximity to the automobile being auctioned may be better able to inspect the car themselves than distant bidders.

Figure 1 attempts to replicate the motivating picture from Laffont, Ossard, and Vuong (1995). However, this replication may have been a mechanical result if the winning bidder tended to be the only bidder bidding above the reserve price. With a bid increment this would have guaranteed that the winning bid and the reserve price moved in lockstep. However, this is not the case because while I do not see any bids other than the winning bid, I do know the number of different bidders bidding on each car and how many were above the reserve price. On average just under five bidders bid on each car and approximately four bid above the reserve price. In fact, I constructed the figure so that no auction in the figure received less than two bids above the reserve price. Therefore, we can be confident that the comovement in winning bids and reserve prices isn't mechanically derived.

The identification and estimation of the model rely on the fact that there is nothing that enters the reserve price equation that does not enter the bidding function. This rules out a seller's private signal or the unobserved heterogeneity affecting only the seller's resale opportunities (or in general his outside option) should he not be able to sell the item at the auction. In regards to the private signal entering the reserve pricing function, as mentioned above, the auction house usually sets the reserve price. This means that it is not the seller who is setting it, and thus it is less likely that there is a private signal entering this function which varies from car to car. In terms of the unobserved heterogeneity entering the reserve pricing function, but not the bidding function, one could imagine, for example, that the seller is an individual looking to acquire a new car once he sells his current one. If the seller derived more utility from driving a car with less dents, say, than a similar one with more dents, then it is conceivable that the seller would wish to set a higher reserve price for a car with less dents although the bidders might not value the car any differently from one with less dents. This seems highly implausible since the bidders in these auctions are themselves used car dealers

\footnotetext{
${ }^{26}$ In fact, bidders are often sent by a downstream purchaser to bid for a certain automobile on the downstream purchaser's behalf, which lends further support to the assumption of independent private values over affiliated private values made in Section 5 .

${ }^{27}$ This auction house was viewed as selling "high quality" used cars so that issues involving "Lemons" and other bidders potentially having information pertinent to one anothers' values may not be as relevant.
} 
concerned with selling the car to buyers who may have preferences similar to the current owner of the car. Nevertheless, we can test the hypothesis that the unobserved heterogeneity only affects reserve prices, and not bidder values, (or more generally that there is something in which reserve prices are increasing but bids are not) by looking at whether the probability of a failed sale due to a lack of bids above the reserve price increases in the reserve price. From the logit model of failure described above, I find that the reserve price is not a statistically, or economically ${ }^{28}$, significant determinant of failure to sell. This lends strong support to the assumption that the unobserved automobile heterogeneity affects both bids and reserve prices.

Another possibility is that sellers have private information about the cars that bidders value. This is similar to the model studied in Lewis (2007). How should the seller's private information affect the seller's reserve price? Cai, Riley, and Ye (2007) develop a model where bidders value the seller's private information and it is incentive compatible for sellers to use their reserve price as a signal of this information: the separating equilibrium is for sellers with higher realizations of $\varepsilon$ to set higher reserve prices. There is a testable implication of this model in the data. Cai, Riley, and Ye (2007) show that when reserve prices can be used as signals that the optimal reserve price is increasing in the number of bidders. Therefore, we can test whether this holds in the data. When we regress the log of reserve prices on automobile's characteristics and the number of bidders there is a positive but insignificant relationship between the number of bidders and the reserve price (p-value of 0.44 ). This, along with the previous evidence that there is not a private signal which affects reserve prices but not bids, provides support for modeling reserve prices as functions of $Z$ and $U$ alone.

The assumption of a single index, as applied in equation 9, would be violated if after controlling for variation in bids by a flexible function of reserve prices, the unexplained variation in bids then could be explained by movement in the observables which ought to have affected reserve prices. To grasp this violation, consider a seller who has the luxury of selling one of two cars. The cars have quite different characteristics, all of which he would observe, but the value of $h(\cdot)$, which to him is a known function, is the same for each car. The assumptions about the forms of $\beta(\cdot)$ and $m(\cdot)$ guarantee that he should be indifferent between selling these two cars. However, if he knew that bidders would still tend to bid more for one of the cars due to the difference in characteristics, then he would not be indifferent between the cars and thus our assumptions about the degree to which variations in cars could be accounted for by this single index $h(\cdot)$ are wrong. ${ }^{29}$ This thought experiment motivates the following test. Calculate the Goodness of fit from the following regression:

$$
B=g(R)+\epsilon_{1}
$$

where $B$ is the winning bid and $g(\cdot)$ is some nonparametric function of reserve prices $R$. Then, run

\footnotetext{
${ }^{28}$ As the reserve price increases from from the 25 th percentile to the 75 th percentile, the probability of failure increases approximately 1 percentage point.

${ }^{29}$ It does not mean that the assumption of $a$ single index is wrong, but rather that we hadn't specified a rich enough single index to satisfy the assumptions about bidder and seller behavior.
} 
the following regression:

$$
B=g(R)+\gamma Z+\epsilon_{2}
$$

where $Z$ is the observable auction covariates, and compare the overall fit of the model to that without the inclusion of $Z$. To perform the test I let $g(\cdot)$ be a fifth order polynomial in the reserve price. The $\mathrm{R}^{2}$ of the first regression is 0.9675 . However, the $\mathrm{R}^{2}$ of the second regression is 0.9695 . This test provides highly supportive evidence that the assumption of a single index in the functions $m(\cdot)$ and $\beta(\cdot)$ is valid since after controlling for reserve prices in a flexible way, the unexplained variation in winning bids is not explained by the automobile's characteristics. ${ }^{30}$

Also, I assume that bidders have unit demands. This is a questionable assumption, though one that is very common in empirical auction work due to its simplifying implications. To justify making the assumption in my data, I note that almost $75 \%$ of the time that a bidder wins a car, he wins less than three cars that day. Moreover, among those days on which a bidder wins two cars, over half the time those cars are of different makes, and when they are of the same make, around $2 / 3$ of the time the car is of different models. This means that the majority of the time the cars being purchased are meeting unique demands of the buyers. There is also evidence of unit demands in Table ?? since the likelihood of failure is increasing in the order in which the vehicle is auctioned within any given day. ${ }^{31}$

Finally, I have assumed throughout that all sellers use the same reserve pricing function $m(\cdot)$. This is potentially problematic since individual sellers are the ones bringing the automobiles to be sold. However, the majority of the time the auction house provides guidance on how to set the reserve price of the car. To the extent that the auction house is an "expert" on the auction market, it then serves as a mechanism for translating car characteristics into reserve prices. Moreover, as the analysis will bear out, it appears that the reserve prices are moving in consistent ways across vehicles despite the fact that the identity of the sellers is changing.

Having built support for the validity of the model's assumptions, I now turn to estimating the distribution of bidder values and evaluating unobserved heterogeneity's impact on policy questions of interest.

\section{Estimation}

In this section I report the results from nonparametrically estimating the distribution of bidder values based on the single index modeling specification that results in the bidding function in equation 10. I also explore how ignoring unobserved heterogeneity matters in our counterfactual

\footnotetext{
${ }^{30}$ However, an F-test of $\gamma=0$ produces a test statistic of 12.81 thus rejecting the coefficients all jointly being zero. Still, I will rely on the extremely small increase in the $R^{2}$ as support for the single index assumption.

${ }^{31}$ Although not shown here, I attempted to test whether the cars were ordered according to some characteristic by regressing the auction order within a day on the car's characteristics for the period of time I have both the failed and successful sales, and it does not seem that there is any statistically significant relationship between when a car is auctioned and its characteristics. One caveat is that Daewoo automobiles seem to be auctioned off first, but this is not likely to be a problem since most dealers purchase two or less cars per day and demands for cars of different makes are likely to be distinct.
} 
experiments. I model the automobiles as being sold through a symmetric IPV English Button auction. It is well known that in these auction potential buyers bid their values.

To begin, I focus on certain kinds of cars with characteristics $Z$. Then, within this collection of cars, I further segment by reserve price. I wish to estimate the distribution of bidder values for these cars at each reserve price, say $R$. In order to do so I need to specify the number of potential bidders for this car. This is because the winning bid is the second highest among $N$ bidders from the true distribution of values. Below, I outline how I specify the number of potential bidders, but for now, suppose we know that this is $N$. Then, I can relate the distribution of winning bids for these auctions to the distribution of values for these cars according to the following mapping. Let $v_{(i: N)}$ be the $i^{\text {th }}$ highest of $N$ draws from $F_{V}$ and denote its distribution by $F_{V_{(i: N)}}$. From the bidding equilibrium above, we know that if $F_{W \mid N}$ is the distribution of winning bids across auctions with $N$ potential bidders, then $F_{W \mid N}=F_{V_{2: N}}$. Furthermore we know that (see Athey and Haile (2002) and more generally Arnold, Balakrishnan, and Nagaraja (1992).)

$$
F_{V_{(i: N)}}(v)=\frac{N !}{(N-i) !(i-1) !} \int_{0}^{F_{V}(v)} u^{N-i}(1-u)^{i-1} d u
$$

By observing the winning bid we are actually observing $v_{(2: N)}$ and 15 allows us to nonparametrically map a distribution of winning bids to $F_{V}(v)$.

The estimation technique is a two step procedure. First $F_{V_{(2: N)}}(v)$ must be obtained from $F_{W}(v)$. No longer suppressing notation, I use the nonparametric kernel estimator for this distribution from Nadaraya (1964) which is given by:

$$
\widehat{F}_{W \mid \tilde{Z}=\tilde{z}}(v)=\frac{\sum_{t=1}^{T} \tilde{k}_{1}\left(\frac{v-W^{t}}{\sigma}\right) k_{2}\left(\frac{\tilde{z}-\tilde{Z}^{t}}{\sigma}\right)}{\sum_{t=1}^{T} k_{2}\left(\frac{\tilde{z}-\tilde{Z}^{t}}{\sigma}\right)}
$$

where $\sigma$ is the bandwidth, $k_{1}: \Re \rightarrow \Re$ and $k_{2}: \Re^{L} \rightarrow \Re$ are some kernel functions and $\tilde{k}_{1}=$ $\int_{\infty}^{u} k_{1}(s) d s$.

Matzkin (2003) shows that under certain regularity conditions, $\widehat{F}_{W \mid \tilde{z}=\tilde{z}}(v)$ is asymptotically normal with mean $F_{W \mid \tilde{Z}=\tilde{z}}(v)$ and variance $V_{F_{W}}$. The next proposition derives the asymptotic distribution of the estimator for the distribution of values, $\widehat{F}_{V \mid \tilde{Z}=\tilde{z}}(v)$.

Proposition 3. Let the number of potential bidders in $T$ auctions be a constant $N$. Let $F_{V \mid \tilde{Z}=\tilde{z}}(v)$ be the true distribution of bidder values conditional on $\tilde{Z}=\tilde{z}$. Then,

$$
\sqrt{T} \sigma_{T}^{(L / 2)}\left[\widehat{F}_{V \mid \tilde{Z}=\tilde{z}}(v)-F_{V \mid \tilde{Z}=\tilde{z}}(v)\right] \rightarrow N\left(0, V_{F_{V}}\right)
$$

where,

$$
V_{F_{V}}=\frac{V_{F_{W}}}{N^{2}(N-1)^{2} F_{V \mid \tilde{Z}=\tilde{z}}(v)^{2(N-1)}\left(1-F_{V \mid \tilde{Z}=\tilde{z}}(v)\right)^{2}}
$$

To test hypotheses, we need to replace $V_{F_{W}}$ and $V_{F_{V}}$ with consistent estimators, which under the assumptions of Proposition 3 can be done by replacing the conditional distributions with their 
kernel estimators.

However, to pool across auctions with different numbers of actual bidders I need to assume that the number of potential bidders is constant across auctions. Unfortunately, the number of potential bidders is unobservable. There are methods for avoiding this complication based on the techniques outlined in Song (2004), but they require having data on more than one bid and also being able to interpret all bids used in the estimation procedure as order statistics. Since I only have data on the winning bid, I am forced to take a stand regarding the number of potential bidders in an auction. ${ }^{32}$ One way to detect how many potential bidders may be present is to count the number of bidders that actually place a bid at some point during the auction, regardless of whether this is the winning bid or not. Yet, even this number is likely to understate how many potential bidders there really are since some potential bidders may not bid. Therefore, I will take as the number of potential bidders the maximum of the number of bidders bidding in any one auction. However, to lend this assumption more credence, I will only pool similar auctions across auction days if the number of bidders showing up at the auction house is similar on these days. In looking at the data, there is not much variation of unique winners within any month, but there is quite a bit of variation across months as evidenced in Table 6. Regardless, these results support further partitioning the data into three categories based on number of potential bidders. Based on the month in which the auction takes place, I assign it to one of these groups and within each group I assume that the same number of potential bidders are present. The groupings by month are given in the following table: ${ }^{33}$

\begin{tabular}{|c|c|c|c|c|c|c|c|c|c|}
\hline Group & & & & & Month & & & & \\
\hline A & $11 / 02$ & & & & & & & & \\
\hline B & $10 / 01$ & $11 / 01$ & $12 / 02$ & $1 / 02$ & $5 / 02$ & $7 / 02$ & $8 / 02$ & $9 / 02$ & $10 / 02$ \\
\hline $\mathrm{C}$ & $2 / 02$ & $3 / 02$ & $3 / 02$ & & & & & & \\
\hline
\end{tabular}

\subsection{Estimation Results}

I now present my estimation results. For the time being, I focus only on five passenger Hyundai Sonatas with automatic transmissions that sold during the months in category B above. For these figures I choose four combinations of mileage and age. Then for each of these four combinations, I choose four reserve prices that are the 15th, 25th, 50th and 90th percentiles of reserve prices for

\footnotetext{
${ }^{32}$ An alternative might be to specify the distribution of potential bidders, say a Poisson distribution, and assume that the number of actual bidders are draws from this distribution. This technique is used in Bajari and Hortaçsu (2003).

${ }^{33}$ Revisions to the table were tried with minor implications for the empirical results. One potential change is to break group B into two groups, one for late 2001, early 2002 and the other for mid-late 2002 to allow for any sector wide changes in demand. However, a Kolmogorov-Smirnov test of the equality of the distributions of winning bids for similar cars in the two groups failed to reject the null hypothesis at the $10 \%$ level.
} 
cars with these mileage and age profiles. ${ }^{34}$ I then specify both of the following models:

$$
\begin{gathered}
B=\beta(\text { MILEAGE, AGE }, S) \\
B=\beta(R, \text { MILEAGE, AGE }, S)
\end{gathered}
$$

and estimate the distribution of values conditional on observables specified to affect bidder behavior. Consider equation 16. Here I attempt to control for all observable heterogeneity by looking only at five passenger Hyundai Sonatas with automatic transmissions that sold during specific months when approximately the same number of bidders showed up at the auction and including the age and mileage of the car as determinants of bids. Therefore, the difference between the two specifications is that the latter utilizes reserve prices to proxy for the unobserved heterogeneity in the cars. To proceed I specify that the number of potential bidders is eight since over $92 \%$ of the auctions have less than this many bidders placing bids above the reserve price.

To begin understanding the output of the estimation, consider Figure 2. The "flatter" distribution labeled "All Cars" is the estimated distribution of values for 8.5 year old cars with 150,000 KMs when I do not control for unobserved heterogeneity. The dashed lines are $95 \%$ confidence intervals. The values are in logs, so indeed there is a great variation in them even within this transmission-make-model-passenger-mileage type of car.

The other four distributions in Figure 2 attempt to control for unobserved heterogeneity. In addition to age and mileage, these four distributions also control for the secret reserve price of the Hyundai Sonatas. There is a clear ordering of the distributions in a stochastic dominance sense: value distributions for cars with higher secret reserve prices maintain a first order stochastic dominance over those with lower reserve prices. This coincides with our intuition that whatever the econometrician is not observing, the more favorable is this characteristic, the higher ought to be both the bids and the reserve prices. Also, there is very little overlap in the distributions and for most of the mass of the values, the distributions are fairly precisely estimated.

Percentiles of the estimated distribution of bidder values appear in Table 7. In this table "Fvall" refers to the distribution of values not controlling for unobserved heterogeneity. The distributions depend greatly on controlling for unobserved heterogeneity as the median of the value distribution may rise more than $500 \%$ depending on its realization.

In Figure 2 the distribution labeled "All Cars" appears to maintain a second order stochastic dominance over the distributions controlling for unobserved heterogeneity despite the fact that even in the distribution entitled "All Cars", I have controlled for a great deal of variation: the make, model, transmission, mileage, age, number of passengers and the time of the year. This suggests that the reserve price is helping soak up variation in bids that otherwise we would have attributed to variation in bidder values. This coincides with our intuition that not controlling for unobserved heterogeneity attributes too much variation in bids to variation in bidder values. To illustrate this

\footnotetext{
${ }^{34}$ In practice I allow cars with "similar but not exactly" these covariates to enter the estimation by smoothing over the data using a Gaussian kernel with approximately the standard choice of bandwidth of $1.06 \sigma T^{\frac{1}{5}}$, where $\sigma$ is the sample standard deviation of the covariate suggested in Silverman (1986).
} 
point, I calculate the interquartile ranges for each of the distributions. The results appear in Table 8. It is clear that the estimated distributions are much more "condensed" when we control for unobserved heterogeneity, although there doesn't seem to be much dependence of the interquartile range on the level of unobserved heterogeneity. However, when I calculate the coefficients of variation of the distributions, the greater the age and mileage of the car, the greater is the percentage reduction in coefficients of variation when we control for unobserved heterogeneity. The percentage reduction for the lowest and highest age and mileage profile is approximately $11 \%$ and $50 \%$ respectively. The degree to which the first and second order stochastic dominance relationships between the estimated value distributions play a role in the outcome of counterfactual experiments of interest will be explored below.

The distributions for the other age-mileage profiles appear in Figure 3 where we see similar results to those highlighted above.

Before moving on to two counterfactual experiments of interest, I explore how unobserved heterogeneity impacts the estimation of two objects of interest in auctions: (a) within-auction variation in bidder values and (b) the surplus captured by bidders.

\subsection{Controlling for Within-Auction Variation}

Having seen that reserve prices help control for unobserved heterogeneity, I now investigate the extent to which their inclusion can alleviate a lack of data when estimating within-auction variation in bids. Since it is common for second price or English auction data sets to include only transaction prices, we might wonder whether controlling for the reserve price of the item can help estimate the amount of variation in bids for similar items, something that would be much more easily assessed had we data on all bids within each auction. Aside from generally addressing the penalty for not having multiple bids, these estimates may be of particular value for risk averse sellers. To analyze this potential use of reserve prices, I use the smaller data set mentioned above which has all bids in an auction during the last three months of 2001.

To proceed, I select similar automobiles and analyze (i) the average within-auction variation not controlling for reserve price, (ii) the across-auction variation not controlling for reserve price and (iii) the across-auction variation controlling for reserve price. By controlling for the reserve price, we can determine how much of the across-auction variation is accounted for as compared to when we have data on all bids within each auction. The results of such a test appear in Table 9 . This table focuses on five passenger Hyundais with automatic transmissions that sold during the last three months of 2001. It divides these cars into 16 milege/age categories defined by the quantiles of each covariate. For each category it computes the within and across-auction variation in bids. Then, for each mileage/age category, it separates the cars into four reserve price categories and computes the across-auction variation in bids. First, the table clearly shows the penalty for not observing multiple bids in an auction when estimating within-auction variation as the numbers in the top row of the table are much smaller than those in the second row. However, a great deal of the variation in the second row can be "soaked up" by controlling for the reserve prices. On average, ignoring 
the last row which appear to be greatly affected by outliers, controlling for the reserve price reduces the across-auction variation in bids by $75 \%$. This suggests that when economists face the frequent challenge of estimating bid variation using only transaction prices, controlling for reserve prices can ameliorate the situation a great deal.

\subsection{Measuring Bidder Surplus}

One consequence of failing to account for unobserved heterogeneity when estimating the distribution of bidder values is that too much variation in bids is attributed to variation in bidder values. This leads to an overestimate of the "dispersion" of the distribution of bidder values. In this subsection I show that this leads to an upward bias in the estimate of the surplus accruing to bidders and then I quantify this overestimate in the data.

An overstatement of the dispersion in bidder values will lead to an upward bias in the estimate of bidder surplus. To see this, consider any auction with $N$ bidders with values drawn from the distribution $F$. Let the winning bid in the auction be denoted $w$ with density $g(w \mid N)$ when there are $N$ bidders. Then, in an English button auction, the expected surplus obtained by the winning bidder, $S(F)$, is given:

$$
\begin{aligned}
S(F) & =\int_{\underline{v}}^{\bar{v}} v_{(N: N)} f_{(N: N)}(v \mid N) d v-\int_{\underline{v}}^{\bar{v}} w g(w \mid N) d w \\
& =\int_{\underline{v}}^{\bar{v}} v_{(N: N)} f_{(N: N)}(v \mid N) d v-\int_{\underline{v}}^{\bar{v}} v_{(N-1: N)} f_{(N-1: N)}(v \mid N) d v
\end{aligned}
$$

To compare the estimate of the surplus captured by bidders when we do and do not control for unobserved heterogeneity, I need to make a useful assumption regarding the relationship between the two estimated value distributions. In the following proposition I assume that the difference between any two quantiles of the estimated distribution of bidder values which controls for unobserved heterogeneity is less than that from the estimated distribution of values which ignores unobserved heterogeneity. While this may not have to be true, it is a useful way to formalize the distinction between these estimated distributions which seems to hold in this paper as well as in others (Krasnokutskaya (2009)).

Proposition 4. Let $F$ and $G$ be two distributions of bidder values such that:

$$
F^{-1}(d)-F^{-1}(c) \leq G^{-1}(d)-G^{-1}(c), \forall 0<c \leq d<1
$$

In an English auction consisting of $N$ bidders, the estimated surplus accruing to bidders based on distribution $F$ will be smaller than that based on distribution $G$.

To test whether the proposition holds in the data, I determine the estimated distribution of bidder values for each age and mileage profile, ignoring unobserved heterogeneity, and then conditioning on its median realization I estimate the bidder surplus for each distribution by simulating 5000 auctions consisting of 3,5 and 10 bidders. The comparison of the estimated surplus accruing 
to the winning bidder for each value distribution appears in Table 10. While the strict quantile dominance assumption used in Proposition 4 may not hold for these distributions, it is evident that failing to control for unobserved heterogeneity leads to an upward bias in the estimated bidder surplus, sometimes by as much as $40 \%$.

\subsection{Reserve Price Setting and Profit Evaluation}

Reserve prices play an historically important role in the empirical auction literature as they were some of the original policy variables of interest in such work as Paarsch (1997). To see what implication not controlling for unobserved heterogeneity has for a common policy variable of interest, I now investigate how an advisor's suggested reserve price would change based on whether the estimated distribution of values affecting the optimal reserve price controlled for unobserved heterogeneity. According to Equation 2, if we had values for $\mathrm{v}_{0}$, then we could estimate the optimal reserve price both controlling and not controlling for unobserved heterogeneity. Unfortunately, I do not have any estimates for $\mathrm{v}_{0}$, so I will simply estimate the optimal reserve prices for a variety of reasonable values for $\mathrm{v}_{0} \cdot{ }^{35}$

The estimated optimal reserve prices for each age-mileage profile and realization of unobserved heterogeneity, $\widehat{\rho}$, for various values of $\mathrm{v}_{0}$, appear in Table 11. Ignoring the column which does not control for unobserved heterogeneity, we see that within any value for $\mathrm{v}_{0}$, and within any reserve price, as the age-mileage profile of the car goes up, the optimal reserve price falls, a sensible result. Also within any value for $\mathrm{v}_{0}$, and within any age-mileage profile, as the reserve price category, or unobserved heterogeneity value, of the car goes up, the optimal reserve price rises. ${ }^{36}$ This confirms that the necessary restrictions in proposition 1 hold in the data. Also evident in Table 11, the estimated optimal reserve price ignoring unobserved heterogeneity is always greater than the estimated optimal reserve price for the median realization of unobserved heterogeneity. This is due to the greater dispersion in the estimated value distribution ignoring unobserved heterogeneity.

What implication does the variation in these "optimal" reserve prices have for expected outcomes of interest to a hypothetical seller? At the median realization of unobserved heterogeneity, the seller that fails to correctly set the optimal reserve price due to ignoring unobserved heterogeneity suffers a loss of around $5 \%$ of revenues. Greater losses result from more extreme realizations of unobserved heterogeneity. Part of the drop is due to an increase in the failure to sell since the optimal reserve price will tend to be too high when unobserved heterogeneity is ignored. At the median realization of unobserved heterogeneity, cars may be twice as likely to fail to sell when the reserve price is set ignoring unobserved heterogeneity, depending on the seller's value for the car.

There are a few caveats to the results on optimal reserve prices. The first is that one might think that $\mathrm{v}_{0}$ should be a function of the characteristics of the car. That is, a low $\mathrm{v}_{0}$ is likely to be more

\footnotetext{
${ }^{35}$ A similar technique is used in Haile and Tamer (2003). As noted there, unlike the requirement in Myerson (1981) that the inverse hazard rate is strictly monotone in $\rho$, not $U$, this procedure only requires that $\Pi\left(\rho ; \mathrm{v}_{0}, F_{V}\right)$ is strictly pseudo-concave on $\mathrm{A} \subseteq \Re$ which means that for all distinct $a_{1}$ and $a_{2} \in \mathrm{A}$, if $\Pi^{\prime}\left(a_{1}\right)\left(a_{2}-a_{1}\right) \leq 0$, then $\Pi\left(a_{2}\right)-\Pi\left(a_{1}\right)<0$. See Avriel, Diewert, Schaible, and Zang (1988).

${ }^{36}$ This in itself is another validation of the model since given its assumptions one should find the optimal reserve price increasing in $U$. I thank Phil Haile for highlighting this result.
} 
appropriate for a car with age-mileage profile 4 than 1 . The second caveat is that the results in these tables are based on estimations of equation 2, which assumes that the seller is a monopolist selling a single good. This is not likely to be the case regardless of whether one considers the seller to be the auction house or the individual car owners who bring their automobile to the auction house to sell. The interpretation of the auction house as the seller is problematic because of the competition mentioned above and it is not selling one good since it frequently sells similar cars on the same day. Interpreting the individual car owners as the seller is problematic because they are unlikely to have much market power. Nevertheless, the results give a benchmark for the implications for reserve price recommendation when the advisor fails to account for unobserved heterogenetiy. Moreover, this benchmark is relevant since there are examples where economists have treated the optimal reserve pricing problem as though it were one faced by a (i) profit maximizing (ii) monopolist who is (iii) selling a single good when in fact one or more of these assumptions may be false.

\subsection{Price Discrimination with Unobserved Heterogeneity}

It is well known that a firm could increase its profits if it could price discriminate instead of charging a uniform price to all consumers. However, this ignores the firm's costs of undertaking such price discrimination. For example, it may be difficult to evaluate its customer types in order to generate a personalized price. That is, the presence of heterogeneity across automobiles, which is unobservable to the auction house without further inspection, creates learning costs that may force it to use a system akin to a two-part tariff in order to approximate perfect price discrimination. The auction house considered in this paper is not unique. Other auction platform operators utilize a similar fee structure. Christie's and Sotheby's, for example, charge 15\% - 20\% of the item's final selling price but do not require flat fees upfront. eBay charges a fixed fee to post an item and then collects a commission of approximately $8.75 \%$ of the winning bid. See Lucking-Reiley (2000) for more details. In this section I evaluate how well the auction house's current pricing scheme captures its customers' surplus in comparison to a scenario where it could perfectly price discriminate. I will use my estimates of the distribution of bidder values to answer this question. First, I show that under a general set of preferences and the assumption that the auction house occurs no cost in price discriminating, that it would be better off eliminating the commission and charging each seller a fixed fee based on the car type. Next, I assume that the auction house's use of a commission and a constant fixed fee for all cars is optimal when faced with the costs of undertaking such a pricing scheme. This allows me to pin down the primitives of the seller's demand for the auction house. Using this parameterized demand, I estimate the optimal fixed fee for each car type. Finally, I assess the maximum of the potential gains per car from price discriminating as compared to the two-part tariff used by the auction house. In this way the section is related to the work in Chu, Leslie, and Sorensen (2008) which evaluates the efficiency of less-than-optimal, but also easier-to-implement, nonlinear pricing schemes in theater ticket sales.

To examine the impact of altering the auction house's commission $t$ and fixed fee $F$, I need to consider how each side of the market responds to a change in these prices. If we assume that 
the seller pays all of the commission, which seems reasonable given that bidders are bidding their true values, then when the auction house raises the commission, the effective values faced by the seller are lower. Suppose that with no commission that the distribution of bidder values is given by $F_{v}$. Then with a commission $t$, the effective distribution of bidder values is given $F_{\widetilde{v}}$ such that $F_{\widetilde{v}}(a)=F_{v}\left(\frac{a}{1-t}\right)$. Therefore, for any constant outside value of the car to the seller, as the auction house raises the commission, the seller faces a less favorable distribution and so he will lower his reserve price. His lowering the reserve price will alter the probability of sale. If $r_{0}$ is the reserve price with no commission and if $r_{t}$ is the reserve price when the commission is $t$, then as long as $r_{t}>(1-t) r_{0}$, the probability of sale will fall since:

$$
\operatorname{Pr}(\text { Sale } \mid t=0, N)=\operatorname{Pr}\left(\max _{i=1, \ldots, N}\left(v_{i}\right)>r_{0}\right)
$$

and:

$$
\begin{aligned}
\operatorname{Pr}(\text { Sale } \mid t>0, N) & =\operatorname{Pr}\left(\max _{i=1, \ldots, N}\left((1-t) v_{i}\right)>r_{t}\right) \\
& =\operatorname{Pr}\left((1-t) \max _{i=1, \ldots, N}\left(v_{i}\right)>r_{t}\right) \\
& >\operatorname{Pr}(\text { Sale } \mid t=0, N) \text { if } r_{t}<(1-t) r_{0} \\
& <\operatorname{Pr}(\text { Sale } \mid t=0, N) \text { if } r_{t}>(1-t) r_{0}
\end{aligned}
$$

I do not assume that sellers set reserve prices optimally, but if I did require this, the next proposition shows that as $t$ increases, the reserve price falls but that $r_{t}>(1-t) r_{0}$ implying that the probability of failure increases. Note that the condition that the hazard rate is decreasing is a sufficient but not necessary condition for the proposition to hold.

Proposition 5. Let $\frac{1-F_{v}(x)}{f_{v}(x)}$ be decreasing in $x$. Let $r_{0}$ and $r_{t}$ be the optimal reserve price in an English button auction set by the seller when the commission is 0 and $t$ respectively. Then $(1-t) r_{0}<r_{t}<r_{0}$.

While sellers will lower their reserve price as the commission increases, they will not alter their reserve price in response to a change in the fixed fee because it does not impact the effective distribution of values they face. While it is possible that bidding behavior is affected by the impact on reserve prices, I assume below that bidding behavior is unchanged when the commission is altered.

I now analyze the optimal pricing decision of the auction house and show that if there were no cost in price discriminating based on type of car, it would be optimal for it to charge 0 commission and a car-type specific fixed fee. Currently the auction house charges anyone wishing to sell their car $\$ 50$. Then, if the car sells, it collects $4.4 \%$ of the winning bid as a commission. Therefore, if we model the seller as bearing the full $4.4 \%$, the effective price paid by the sellers is:

$$
P(t, F)=t \pi(t) W+F
$$

where $W$ is the expected winning bid for the car at the auction house, $\pi(t)$ is the probability that the 
car sells at a given commission $t$ and $F$ is the fixed fee. ${ }^{37}$ The probability of sale decreases in $t$ based on the argument above. Let the expected gross revenue earned by the seller be $R(t, F)=\pi(t) W$ and define $\widetilde{P}(t, F)=R(t, F)-P(t, F)$ as the expected cash flow of the seller who chooses the auction house.

I now model the sellers' decision of whether or not to use the auction house. Consider the seller of a given car. Suppose he has outside option $\varepsilon \sim G(\varepsilon)$ with associated density function $g(\cdot)$. Let the seller's preferences over bundles of probability of sale and prices $(\pi(t), \widetilde{P}(t, F))$ be: ${ }^{38}$

$$
U(\pi(t), \widetilde{P}(t, F))=\pi(t)+\widetilde{P}(t, F)+(1-\pi(t)) \varepsilon
$$

For a given commission and fixed fee, a seller will choose the auction house provided $U(\cdot)>\varepsilon$.

Suppose that the firm has 0 marginal costs and a fixed cost of operating equal to $\phi$. Then the firms profit function is given:

$$
\Pi(t, F)=P(t, F) G(U(\pi(t), \widetilde{P}(t, F)))-\phi
$$

I will now argue that if the auction house has full information regarding a seller's car, it should only charge the seller a fixed fee and set the commission to zero. The intuition for the argument is that $t$ distorts seller decisions at the margin and hence reduces total surplus. Therefore, it is optimal for the auction house to set a fixed fee to extract expected surplus. To see this, note that the tradeoff between $t$ and $F$ that holds the seller indifferent is:

$$
\frac{d F}{d t} \bar{U}=\frac{1}{\pi(t)} \frac{\partial \pi(t)}{\partial t} P(t, F)-\left(\pi(t) W+t \frac{\partial \pi(t)}{\partial t} W\right)
$$

and that the tradeoff between $t$ and $F$ that holds constant the price charged by the auction house:

$$
\frac{d F}{d t} \bar{P}=-\left(\pi(t) W+t \frac{\partial \pi(t)}{\partial t} W\right)
$$

so that:

$$
\frac{d F}{d t}=\frac{1}{\pi(t)} \frac{\partial \pi(t)}{\partial t} P(t, F)+\frac{d F}{d t} \bar{P}
$$

\footnotetext{
${ }^{37}$ Equation 19 assumes that the $\$ 50$ fee is nonrefundable. This is valid if the owner of a car which failed to sell brings the automobile back to the auction house later after taking it home since he will have to pay the $\$ 50$ again. In reality, if the owner decides to leave the car with the auction house after it failed to sell, he would not have to pay the $\$ 50$ at the next auction. The above formulation ignores this possibility. To get a sense of how often sellers leave a car which failed to sell at the auction house, I look for cars which failed to sell one week and are then put up for auction the next week. To do so, however, I identify cars based on their characteristics since there is no unique identifier in the data. This process reveals that during the sample period for which I have failed sales, only $1.07 \%$ of the cars which failed to sell were left at the auction house until the next auction took place. This is based on the mileage of the car being the same week after week, suggesting that the owners didn't take the car home only to return the following week.

${ }^{38}$ This specification can be thought of as a first order Taylor expansion of the seller's utility $U=\pi(t) u((1-t) W)-$ $F+(1-\pi(t)) \varepsilon$ about the risk neutral payoff for the seller if we normalize $u((1-t) W)=(1-t) W$.
} 
Since $\frac{\partial \pi(t)}{\partial t} \leq 0$, as long as $\pi(t) W+t \frac{\partial \pi(t)}{\partial t} W \geq 0,{ }^{39}$ then $\left|\frac{d F}{d t} \bar{U}\right| \geq\left|\frac{d F}{d t} \bar{P}\right|$. Thus, to hold consumers indifferent to a drop in $t$, the firm is able to raise $F$ by more than the amount needed to hold $P(t, F)$ constant. Therefore, it would seek to lower $t$ as much as possible, i.e. set $t=0 .{ }^{40}$

For the moment, let $U(t, F)=U(\pi(t), \widetilde{P}(t, F))$. If the firm sets $t=0$, the optimal fixed fee is given:

$$
F^{*}=\frac{G\left(U\left(0, F^{*}\right)\right.}{g\left(U\left(0, F^{*}\right)\right)}
$$

As long as $\frac{G\left(U\left(0, F^{*}\right)\right.}{g\left(U\left(0, F^{*}\right)\right)}$, also known as the inverse of the reverse hazard rate of $G(\cdot)$, is increasing in $W$, then $F^{*}$ will be increasing in $W$. While the distribution of sellers' outside option is unknown, we can infer from the auction house's use of a flat fee and a commission which is a percentage of the winning bid, that these distributions are characterized by an increasing inverse of the reverse hazard rate in $W$.

I have now shown that the auction house would be better served by charging $0 \%$ commission and a flat fixed fee which varies by car. In reality they do not do this, due to the cost of undertaking such price discrimination. In the face of these costs, I assume that their decision to set a $4.4 \%$ commission and $\$ 50$ flat fee to each seller, regardless of vehicle type, is optimal, allowing me to uncover the parameters of the model from the first order conditions. To do this, I will have to make some strong assumptions.

I will assume that for any car type $j$, the outside option of a seller is given:

$$
\varepsilon_{j}=\gamma W_{j}+\delta \sigma_{j} \eta \sim N\left(\gamma W_{j}, \delta^{2} \sigma_{j}^{2}\right)
$$

where $W_{j}$ and $\sigma_{j}$ are the mean and standard deviation of the winning bid for car type $j$ at the auction and $\eta$ is an i.i.d. standard normal random variable. The restriction is that $\gamma$ and $\delta$ are constant across $j$. The first order conditions evaluated at $t=4.4 \%$ and $F=\$ 50$ then determine $\gamma$ and $\delta$. Though I condition on age and mileage and reserve price when I control for unobserved heterogeneity, I follow the model outlined in Section 3.2 and pool across car types to estimate the distribution of bidder values. To do so, I estimate the distribution of bidder values for every 5 th percentile of age and mileage when I ignore unobserved heterogeneity and every 10th percentile of reserve price within any age and mileage level when I control for unobserved heterogeneity. When I divide the cars sold by the auction house in this way, these parameterizations deliver the results in Table 12.

With these parameters in hand, I can now perform the counterfactual exercise of estimating the optimal fixed fee in the absence of costs to price discriminating. Before moving on to the estimation results, I must specify how sellers alter their reserve prices as a function of $t$. Since I do not observe different values of $t$ in the data, and since I wish to avoid assuming that sellers optimally set their reserve prices, I cannot identify how reserve prices vary with $t$. I assume, therefore, that reserve prices are held constant as $t$ varies but that they are set relative to the winning bid net of

\footnotetext{
${ }^{39}$ This condition holds in the data used in the analysis.

${ }^{40}$ Yao and Mela (2008) find that an online auction company could increase its revenues by reducing commissions.
} 
commission. ${ }^{41}$ This insures that as the commission increases, the probability of sale falls.

Table 12 shows the results for these exercises. It indicates that when ignoring unobserved heterogeneity, there tends to be less variation in the optimal fixed fees as compared to when we control for unobserved heterogeneity. Figure 4 displays the optimal fee structure when controlling for unobserved heterogeneity by fitting a 5th order polynomial to the estimated optimal fees and comparing it to the current two-part tariff used by the auction house. First, we can see why the current pricing scheme is used: the two-part tariff approximates the increasing nature of the optimal fixed fees in the expected winning bid of the cars. Second we can see that the current pricing scheme subsidizes the sellers of more expensive automobiles by "overcharging" the sellers of less expensive cars. This may make sense when aspects of the model which were abstracted from above, such as the reputation effects associated the auction house's repeat purchasers, are taken into consideration. In terms of measuring the potential gains from price discrimination, the bias of ignoring unobserved heterogeneity in this analysis is as expected: controlling for unobserved heterogeneity allows the auction house to more perfectly price discriminate and therefore extract more of the seller's surplus. The magnitude of this bias, however, demonstrates that ignoring unobserved heterogeneity can substantially reduce the estimated potential gains from price discriminating, here by over $70 \%$. The potential gains from price discriminating can be compared to the current average revenue per car of $\$ 165.08$. Taken together, these results suggest that while the auction house's current pricing scheme may approximate the increasing nature of the optimal fixed fees, it may be forgoing over $6 \%$ of revenue by not price discriminating, and potentially a much greater percentage of profits, and these losses would be substantially underestimated if we ignored unobserved heterogeneity.

\section{Conclusion}

The existing empirical auction literature has improved our understanding of how a variety of auctions operate in practice and has answered questions of practical importance such as revenue maximizing design, the detection of collusion and the estimation of firm markups. Much of this literature is hampered, however, by ignoring unobserved heterogeneity. While several recent attempts have been made to combat this outstanding empirical issue, the current methods sometimes impose potentially unpalatable modeling assumptions and strict data requirements.

The current paper proposes a new methodology which relies on using information from the behavior of individuals other than bidders (who are the only auction participants currently used in efforts to control for unobserved heterogeneity), namely sellers, to help control for what the researcher doesn't observe in the data. Oftentimes such seller behavior is available. Though this paper suggests the use of reserve prices, similar seller choice variables such as engineer estimates in procurement auctions or the decision of when to hold an auction, say on eBay, might also be employed. The solution relies on a strict monotonicity assumption in the seller behavior which is intuitively plausible and, while not required in the model, generally is the outcome of optimal

\footnotetext{
${ }^{41}$ I also experimented with parameterizing the reserve prices as functions of $t$. In particular I used the function $r(t)=-k \arctan (t)+1$ for different values of $k$ with no real changes to the results.
} 
seller behavior. While I propose a general nonparametric solution to the problem, I also present a common single index assumption to reduce dimensionality problems. After deriving its asymptotic distribution, my nonparametric estimator of the distribution of bidder values in used car auctions clearly shows the severity of ignoring unobserved heterogeneity.

Finally, I demonstrate several applications and consequences of utilizing reserve prices to control for unobserved heterogeneity. First, reserve prices are shown to be particularly useful when attempting to determine within-auction variation in bids when only single bids per auction are available as data. I show that utilizing reserve prices allows us on average to reduce estimates of the within-auction variation in bids by an average of $75 \%$. Second, I show that the previously held belief that a failure to control for unobserved heterogeneity leads to an overestimate of the variation in bidder values indeed holds in my estimation results. More generally, I show that when this is the case, we will tend to overestimate the amount of surplus accruing to the winning bidder. In my data, ignoring unobserved heterogeneity leads to an overestimate of bidder surplus of approximately $30 \%$. Third, I analyze the ramifications of ignoring unobserved heterogeneity when estimating the optimal reserve price. In my data this can lead to a substantial drop in expected revenues and an increased tendency for the item to go unsold. Fourth, I evaluate the potential gains to the auction house from price discriminating based on a car's attributes. I claim that when there is heterogeneity across vehicle types, which is unobservable to the auction house without incurring learning costs, this will lead the auction house to forgo perfect price discrimination in favor of a two-part tariff. I show that in the absence of such costs, the potential gains from price discriminating are as much as $5 \%$ of revenues and that these gains are underestimated if we ignore unobserved heterogeneity. 


\section{Appendix A: Monte Carlo Study}

In this section I perform a monte carlo study to test the two step estimation procedure. First, I mimic the reserve pricing data by generating data consisting of two covariates, $Z_{0}$ and $Z_{1}$, a random shock $U$ by letting (i) $Z_{0}=z_{0}^{H}$ with probability $p$ and $Z_{0}=z_{0}^{L}$ with probability $1-p$, (ii) $Z_{1} \sim N\left(\mu_{Z_{1}}^{H}, \sigma_{Z_{1}}\right)$ when $Z_{0}=z_{0}^{H}$ and $Z_{1} \sim N\left(\mu_{Z_{1}}^{L}, \sigma_{Z_{1}}\right)$ when $Z_{0}=z_{0}^{L}$ and (iii) $U \sim N\left(\mu_{U}^{H}, \sigma_{U}\right)$ when $Z_{0}=z_{0}^{H}$ and $U \sim N\left(\mu_{U}^{L}, \sigma_{U}\right)$ when $Z_{0}=z_{0}^{L}$. Then, I generate reserve prices by $R=$ $m\left(Z_{0}, Z_{1}, U\right)=Z_{1}\left(\gamma_{0} Z 0+\gamma_{1} Z_{1}\right)+U$ so that $u=m\left(z_{0}, 0, u\right)$ for all $z_{0}$ and $u$ and, by the data generating process, $U$ is independent of $Z_{1}$ conditional on $Z_{0}$. Next, I create a grid of values for $Z_{0}, Z_{1}$ and $U$ that contains $97.5 \%$ of the support of these variables and, for each grid point, I form $R$ according to above function. Then I draw $T$ values of $Z_{0}, Z_{1}$ and $U$ and then compute $R$ according to $m(\cdot)$. For these $T$ data points, I assume that I only would have seen the outcomes $Z_{0}$,

$Z_{1}$ and $R$. I then estimate $F_{U \mid Z_{0}=z_{0}^{H}}, F_{U \mid Z_{0}=z_{0}^{L}}$ and the function $m(\cdot)$ according to the procedure outlined above. Then, I repeat $S$ times. The specifics of the design and the results appear in Table 13. Figure 5 shows that the estimator approximates the true distribution quite well with the true distribution almost always falling within its $95 \%$ bootstrapped confidence intervals.

Now I am interested in evaluating how well performing the first stage estimation can help in estimating the distribution of bidder values when there is unobserved heterogeneity. Based on my first stage estimates of the function $m(\cdot)$, I can recover the realization of unobserved heterogeneity and then simulate $T$ English (or equivalently second price) auctions in which for any auction $t$ there are $N_{t}$ bidders whose values are distributed according to $F$ which depends upon the realization of $U$ according to: $F: \operatorname{Normal}(U \alpha, \sigma)$. Then, assuming only that I have data on the transaction price from these auctions, I attempt to estimate this distribution via maximum likelihood if I know that $V$ is distributed normally and I (i) know the actual realization of the unobserved heterogeneity ("Full Information"), (ii) know that there is unobserved heterogeneity, but must calculate it from the first stage ("Partial Information") and (iii) do not know that there is any unobserved heterogeneity ("No Information"). I estimate the parameters $\alpha$ and $\sigma$ by maximum likelihood where the likelihood function relating transaction prices, $w_{t}$, to the distribution of values in English or second price auctions when the reserve price is not binding, as will be the case in the empirical application, is given:

$$
L=\prod_{t=1, \ldots, T} n_{t}\left(n_{t}-1\right) F\left(w_{t}\right)^{\left(n_{t}-2\right)}\left(1-F\left(w_{t}\right)\right) f\left(w_{t}\right)
$$

I then repeat this process $M$ times. The results appear in Table 14. It is apparent that in the presence of unobserved heterogeneity, while the estimator is outperformed by the scenario where we know the actual realizations of the unobserved heterogeneity, it works a great deal better than ignoring the unobserved heterogeneity all together. 


\section{Appendix C: Proofs}

\section{Proposition 1}

Proof. Affiliation between $V$ and $U$ with joint density $f$ provides that for all $v_{1}<v_{2}$ and $u_{1}<u_{2}$ :

$$
f\left(v_{1}, u_{2}\right) f\left(v_{2}, u_{1}\right)<f\left(v_{1}, u_{1}\right) f\left(v_{2}, u_{2}\right)
$$

Rearranging and making use of the corresponding conditional distributions:

$$
\frac{f\left(v_{2} \mid u_{1}\right) f\left(u_{1}\right)}{f\left(v_{1} \mid u_{1}\right) f\left(u_{1}\right)}<\frac{f\left(v_{2} \mid u_{2}\right) f\left(u_{2}\right)}{f\left(v_{1} \mid u_{2}\right) f\left(u_{2}\right)}
$$

and therefore:

$$
\frac{f\left(v_{2} \mid u_{1}\right)}{f\left(v_{1} \mid u_{1}\right)}<\frac{f\left(v_{2} \mid u_{2}\right)}{f\left(v_{1} \mid u_{2}\right)}
$$

Integrating we have:

$$
\int_{v_{1}}^{\infty} \frac{f\left(v_{2} \mid u_{1}\right)}{f\left(v_{1} \mid u_{1}\right)} d v_{2}<\int_{v_{1}}^{\infty} \frac{f\left(v_{2} \mid u_{2}\right)}{f\left(v_{1} \mid u_{2}\right)} d v_{2}
$$

which yields:

$$
\frac{1-F\left(v_{1} \mid u_{1}\right)}{f\left(v_{1} \mid u_{1}\right)}<\frac{1-F\left(v_{1} \mid u_{2}\right)}{f\left(v_{1} \mid u_{2}\right)}
$$

It is well known that the optimal reserve price for such a seller with cost equal to $\mathrm{v}_{0}$ is defined by Equation 3. Therefore, if $r_{k}^{*}=r^{*}\left(\mathrm{v}_{0}, G, u_{k}\right)$ is the solution when $U=u_{k}, k=1$, , we have $\frac{1-F\left(r_{1}^{*} \mid u_{1}\right)}{f\left(r_{1}^{*} \mid u_{1}\right)}<\frac{1-F\left(r_{1}^{*} \mid u_{2}\right)}{f\left(r_{1}^{*} \mid u_{2}\right)}$ and therefore, since $c(t, U)$ is increasing in $t$ for all $U$, it must be that $r_{1}^{*}<r_{2}^{*}$.

Q.E.D.

\section{Proposition 2}

Proof. Beginning with reserve prices, we can identify the conditional distribution of unobserved heterogeneity as follows: $F_{R \mid\left(Z_{0}, Z_{1}\right)=\left(z_{0}, \overline{z_{1}}\right)}(u)=\operatorname{Pr}\left[R<u \mid\left(Z_{0}, Z_{1}\right)=\left(z_{0}, \overline{z_{1}}\right)\right]=\operatorname{Pr}\left[m\left(Z_{0}, Z_{1}, U\right)<\right.$ $\left.u \mid\left(Z_{0}, Z_{1}\right)=\left(z_{0}, \overline{z_{1}}\right)\right]=\operatorname{Pr}\left[U<u \mid\left(Z_{0}, Z_{1}\right)=\left(z_{0}, \overline{z_{1}}\right)\right]=\operatorname{Pr}\left[U<u \mid Z_{0}=z_{0}\right]=F_{U \mid Z_{0}=z_{0}}(u)$, where the third equality comes from the normalization of $m$ in assumption (i), and the fourth comes from the conditional independence assumption (ii). Also, we note that: $F_{U \mid Z_{0}=z_{0}}(u)=\operatorname{Pr}\left[U<u \mid Z_{0}=\right.$ $\left.z_{0}\right]=\operatorname{Pr}\left[U<u \mid\left(Z_{0}, Z_{1}\right)=\left(z_{0}, z_{1}\right)\right]=\operatorname{Pr}\left[m\left(Z_{0}, Z_{1}, U\right)<m\left(z_{0}, z_{1}, u\right) \mid\left(Z_{0}, Z_{1}\right)=\left(z_{0}, z_{1}\right)\right]=\operatorname{Pr}[R<$ $\left.m\left(z_{0}, z_{1}, u\right) \mid\left(Z_{0}, Z_{1}\right)=\left(z_{0}, z_{1}\right)\right]=F_{R \mid\left(Z_{0}, Z_{1}\right)=\left(z_{0}, z_{1}\right)}\left(m\left(z_{0}, z_{1}, u\right)\right)$, where the second equality again uses independence and the third uses the monotonicity of $m(\cdot, U)$. Therefore, the function $m$ is identified since:

$$
m\left(z_{0}, z_{1}, u\right)=F_{R \mid\left(Z_{0}, Z_{1}\right)=\left(z_{0}, z_{1}\right)}^{-1}\left(F_{R \mid\left(Z_{0}, Z_{1}\right)=\left(z_{0}, \overline{z_{1}}\right)}(u)\right)
$$

Now, with data on reserve prices, $R$, and item characteristics $Z$, due to the strict monotonicity of $m(\cdot, U)$ for each $(R, Z)$ pair there will be a unique realization of $U$ satisfying $R=m(Z, U)$.

Let $W_{0}=[Z, U]$. Using only transaction prices, we can identify $F\left(S \mid W_{0}\right)$ in a first price and second price auction using the arguments of Theorems 6 and 1, respectively, in Athey and Haile 


\section{Proposition 3}

Proof. Let regularity conditions C.1-C.5 from Matzkin (2003), p.1356, hold. From equation ?? we know that $\eta^{-1}\left(\widehat{F}_{W \mid \tilde{Z}=\tilde{z}}(v)\right)=\left(\widehat{F}_{V \mid \tilde{Z}=\tilde{z}}(v)\right)$ where $\eta^{-1}(\cdot)$ is a known continuously differentiable function. Moreover, from Matzkin $(2003)$ we know that $\sqrt{T} \sigma_{T}^{(L / 2)}\left[\widehat{F}_{W \mid \tilde{Z}=\tilde{z}}(v)-F_{W \mid \tilde{Z}=\tilde{z}}(v)\right] \rightarrow$ $N\left(0, V_{F_{W}}\right)$. Let $\xi(\cdot)=\eta^{-1}$ and $\alpha_{T}=\sqrt{T} \sigma_{T}^{(L / 2)}$. Therefore, we can take a first-order, Taylor-series expansion of $\xi\left(\widehat{F}_{W \mid \tilde{Z}=\tilde{z}}(v)\right)$ about $\xi\left(F_{W \mid \tilde{Z}=\tilde{z}}(v)\right)$ to get:

$$
\begin{aligned}
\left.\xi\left(\widehat{F}_{W \mid \tilde{Z}=\tilde{z}}(v)\right)\right)= & \xi\left(F_{W \mid \tilde{Z}=\tilde{z}}(v)\right)+\xi^{\prime}\left(F_{W \mid \tilde{Z}=\tilde{z}}(v)\right)\left[\widehat{F}_{W \mid \tilde{Z}=\tilde{z}}(v)-F_{W \mid \tilde{Z}=\tilde{z}}(v)\right] \\
& +O_{p}\left(\alpha_{T} \frac{\xi^{\prime \prime}\left(F_{W \mid \tilde{Z}=\tilde{z}}(v)\right)}{2}\left[\widehat{F}_{W \mid \tilde{Z}=\tilde{z}}(v)-F_{W \mid \tilde{Z}=\tilde{z}}(v)\right]^{2}\right)
\end{aligned}
$$

Rearranging and multiplying through by $\alpha_{T}$ we get:

$$
\alpha_{T}\left[\xi\left(\widehat{F}_{W \mid \tilde{Z}=\tilde{z}}(v)\right)-\xi\left(F_{W \mid \tilde{Z}=\tilde{z}}(v)\right)\right]=\xi^{\prime}\left(F_{W \mid \tilde{Z}=\tilde{z}}(v)\right) \alpha_{T}\left[\widehat{F}_{W \mid \tilde{Z}=\tilde{z}}(v)-F_{W \mid \tilde{Z}=\tilde{z}}(v)\right]+o_{p}(1)
$$

Therefore, by the continuous mapping theorem and the definition of $\xi(\cdot)$ :

$$
\alpha_{T}\left[\widehat{F}_{V \mid \tilde{Z}=\tilde{z}}(v)-F_{V \mid \tilde{Z}=\tilde{z}}(v)\right] \rightarrow N\left(0, V_{F_{V}}\right)
$$

where:

$$
\begin{aligned}
V_{F_{V}} & =\xi^{\prime}\left(F_{W \mid \tilde{Z}=\tilde{z}}(v)\right) V_{F_{W}} \xi^{\prime}\left(F_{W \mid \tilde{Z}=\tilde{z}}(v)\right) \\
& =\frac{V_{F_{W}}}{N^{2}(N-1)^{2} F_{V \mid \tilde{Z}=\tilde{z}}(v)^{2(N-1)}\left(1-F_{V \mid \tilde{Z}=\tilde{z}}(v)\right)^{2}}
\end{aligned}
$$

and:

$$
V_{F_{W}}=\left\{\int K(u)^{2}\right\}\left[F_{W \mid \tilde{Z}=\tilde{z}}(v)\left(1-F_{W \mid \tilde{Z}=\tilde{z}}(v)\right)\right] / f(\tilde{z})
$$

by Matzkin (2003).

Q.E.D.

\section{Proposition 4}

Proof. This proof is based on work by Shaked and Shanthikumar (1994). Rewriting the supposition, $G^{-1}\left(F\left(v_{1}\right)\right)-v_{1} \leq G^{-1}\left(F\left(v_{2}\right)\right)-v_{2}$ for all $v_{1} \leq v_{2}$. For any collection of $N$ values drawn from a distribution $H$, order them such that $v_{1}^{H} \leq v_{2}^{H} \leq \ldots \leq v_{N}^{H}$, with $v_{(i: N)}^{H}=v_{i}^{H}$. Therefore, the expected surplus in any given English auction with $N$ bidders whose values are thought to be drawn from $F(G)$ will be given by $S(F)=E\left[v_{N}^{F}-v_{N-1}^{F}\right]\left(S(G)=E\left[v_{N}^{G}-v_{N-1}^{G}\right]\right)$. By definition 
$v_{N-1}^{F} \leq v_{N}^{F}$ and so $v_{N}^{F}-v_{N-1}^{F} \leq G^{-1}\left(F\left(v_{N}^{F}\right)\right)-G^{-1}\left(F\left(v_{N-1}^{F}\right)\right)$. Now, the joint distribution of $v_{N}^{G}$ and $v_{N-1}^{G}$ is the same as the joint distribution of $G^{-1}\left(F\left(v_{N}^{F}\right)\right)$ and $G^{-1}\left(F\left(v_{N-1}^{F}\right)\right)$ and therefore $S(F)<S(G)$.

Q.E.D.

\section{Proposition 5}

Proof. Let the seller have an outside value for the car equal to $\mathrm{v}_{0}$. Let $F_{v}$ be the distribution of bidder values when the commission is 0 . The seller, selecting his reserve price in an English button auction when the commission is $t$, will consider himself to be facing values $\widetilde{v}=(1-t) v$. Let the distribution of effective values faced by a seller when the commission is $t$ be $F_{\widetilde{v}, t}$. Note that $F_{\widetilde{v}}(a)=F_{v}\left(\frac{a}{1-t}\right)$ and $f_{\widetilde{v}}(a)=f_{v}\left(\frac{a}{1-t}\right) \frac{1}{1-t}$. The optimal reserve price set by a seller when the commission is 0 is given by:

$$
r_{0}=\mathrm{v}_{0}+\frac{1-F_{v}\left(r_{0}\right)}{f_{v}\left(r_{0}\right)}
$$

When the commission is $t$, the optimal reserve price is given by:

$$
\begin{aligned}
r_{t} & =\mathrm{v}_{0}+\frac{1-F_{\widetilde{v}, t}\left(r_{t}\right)}{f_{\widetilde{v}, t}\left(r_{t}\right)} \\
& =\mathrm{v}_{0}+(1-t)\left(\frac{1-F_{v}\left(\frac{r_{t}}{1-t}\right)}{f_{v}\left(\frac{r_{t}}{1-t}\right)}\right)
\end{aligned}
$$

Recall that by the supposition, $\frac{1-F_{v}(r)}{f_{v}(r)}$ is decreasing in $r$.

Define $\lambda(r) \equiv r-\mathrm{v}_{0}-\frac{1-F_{\widetilde{v}, t}(r)}{f_{\widetilde{v}, t}(r)}$ and note that $\lambda\left(r_{t}\right)=0$. I will now show that $(1-t) r_{0}<r_{t}<r_{0}$. First, I will show that $\lambda\left((1-t) r_{t}\right)<0$ implying that $r_{t}>(1-t) r_{0}$. Then, I will show that $\lambda\left(r_{0}\right)>0$ implying that $r_{t}<r_{0}$. I will occasionally refer to Figure 6 to help demonstrate the intuition.

To show that $r_{t}>(1-t) r_{0}$, note that:

$$
\begin{aligned}
(1-t) r_{0} & =(1-t)\left(\mathrm{v}_{0}+\frac{1-F_{v}\left(\frac{\widetilde{r}}{1-t}\right)}{f_{v}\left(\frac{\widetilde{r}}{1-t}\right)}\right) \\
& =(1-t) \mathrm{v}_{0}+\frac{1-F_{\widetilde{v}, t}\left((1-t) r_{0}\right)}{f_{\widetilde{v}, t}\left((1-t) r_{0}\right)} \\
& <\mathrm{v}_{0}+\frac{1-F_{\widetilde{v}, t}\left((1-t) r_{0}\right)}{f_{\widetilde{v}, t}\left((1-t) r_{0}\right)}
\end{aligned}
$$

Therefore, $\lambda\left((1-t) r_{t}\right)<0$. Graphically, this is shown by comparing point A to point $\mathrm{C}$ in Figure 6. 
To show that $r_{t}<r_{0}$, note that:

$$
\begin{aligned}
\mathrm{v}_{0}+\frac{1-F_{\widetilde{v}, t}\left(r_{0}\right)}{f_{\widetilde{v}, t}\left(r_{0}\right)} & =\mathrm{v}_{0}+(1-t)\left(\frac{1-F_{v}\left(\frac{r_{0}}{1-t}\right)}{f_{v}\left(\frac{r_{0}}{1-t}\right)}\right) \\
& <\mathrm{v}_{0}+\frac{1-F_{v}\left(\frac{r_{0}}{1-t}\right)}{f_{v}\left(\frac{r_{0}}{1-t}\right)} \\
& <\mathrm{v}_{0}+\frac{1-F_{v}\left(r_{0}\right)}{f_{v}\left(r_{0}\right)} \\
& =r_{0}
\end{aligned}
$$

Therefore, $\lambda\left(r_{0}\right)>0$. Graphically, this is shown by comparing point B to the $45^{\circ}$ line in Figure 6 . Thus we have shown $(1-t) r_{0}<r_{t}<r_{0}$, depicted in Figure 6 by point $\mathrm{C}$.

Q.E.D. 


\section{References}

Adams, W., L. Einav, And J. Levin (2007): "Liquidity Constraints and Imperfect Information in Subprime Lending," Working Paper.

Arnold, B., N. Balakrishnan, and H. N. Nagaraja (1992): A First Course in Order Statistics. John Wiley Sons, New York, NY.

Asker, J. (2008): "Subsidizing (and Taxing) Business Procurement," Journal of Public Economics, 92, 1629-1643.

Athey, S., And P. Haile (2002): "Identification of Standard Auction Models," Econometrica, 70, $2107-2140$.

- (2006): Empirical Models of Auctions, vol. 2 of Advances in Economics and Econometrics, Theory and Applications: Ninth World Congress. Cambridge University Press.

Athey, S., J. Levin, and E. Seira (2004): "Comparing Open and Sealed Bid Auctions: Theory and Evidence from Timber Auctions," NBER Working Paper.

Avriel, M., W. E. Diewert, S. Schaible, and I. Zang (1988): Generalized Concavity. Plenum, New York, NY.

Bajari, P., and A. HortaÇsu (2003): "The Winner's Curse, Reserve Prices and Endogenous Entry: Empirical Insights from eBay Auctions," RAND Journal of Economics, 34(2), 329-355.

Bajari, P., And L. Ye (2003): "Deciding Between Competition and Collusion," Review of Economics and Statistics, 85(4), 971-989.

Bulow, J., And J. Roberts (1989): "The Simple Economics of Optimal Auctions," Journal of Political Economy, 97(5), 1060-1090.

Cai, H., J. Riley, And L. Ye (2007): "Reserve Price Signaling," Journal of Economic Theory, $135,253-268$.

Campo, S., I. Perrigne, and Q. Vuong (2003): "Asymmetric Bidders in First-Price Auctions with Affiliated Private Values," Journal of Applied Econometrics, 18, 179-207.

Cassady, R. (1967): Auctions and Auctioneering. University of California Press, Berkeley, CA.

Chu, C., P. Leslie, and A. Sorensen (2008): "Nearly Optimal Pricing for Multiproduct Firms," Working Paper.

Decarolis, F. (2009): "When the Highest Bidder Loses the Auction: Theory and Evidence from Public Procurement," Working Paper. 
Einav, L., And A. Nevo (2007): Empirical Models of Imperfect Competition: A DiscussionAdvances in Economics and Econometrics: Theory and Applications: Ninth World Congress.

Guerre, E., I. Perrigne, and Q. Vuong (2009): "Nonparametric Identification of Risk Aversion in First-Price Auctions Under Exlusion Restrictions," Econometrica, 77(4), 1193-1228.

Haile, P., H. Hong, and M. Shum (2006): "Nonparametric Tests for Common Values In FirstPrice Sealed-Bid Auctions," Working Paper.

Haile, P., And E. TAmer (2003): "Inference with an Incomplete Model of English Auctions," Journal of Political Economy, 111(1), 1-51.

Hong, H., And M. Shum (2002): "Increasing Competition and the Winner's Curse: Evidence from Procurement," Review of Economic Studies, 69, 871-898.

Hu, Y., D. McAdams, and M. Shum (2009): "Nonparametric Identification of Auction Models with Non-Separable Unobserved Heterogeneity".," Working Paper.

Khan, S. (2001): "Two-stage Rank Estimation of Quantile Index Models," Journal of Econometrics, 100, 319-355.

Kim, K., And J. LeE (2006): "A Structural Analysis of Wholesale Used-Car Auctions: Nonparametric Estimation and Testing of Dealers' Valuations," Working Paper.

Kontos, T. (2006): "Vehicle Remarketing and the Dealer," Industry report, ADESA, Inc.

Krasnokutskaya, E. (2009): "Identification and Estimation in Highway Procurement Auctions Under Unobserved Auction Heterogeneity," Review of Economic Studies, forthcoming.

Laffont, J.-J., H. Ossard, and Q. Vuong (1995): "Econometrics of First Price Auctions," Econometrica, 63(4), 953-980.

Lewis, G. (2007): "Asymmetric Information, Adverse Selection and Seller Disclosure: The Case of eBay Motors," Working Paper.

Li, T., I. Perrigne, and Q. Vuong (2000): "Conditionally Independent Private Information in OCS Wildcat Auctions," Journal of Econometrics, 98, 129-161.

Li, T., And Q. Vuong (1998): "Nonparametric Estimation of the Measurement Error Model Using Multiple Indicators," Journal of Multivariate Analysis, 65, 139-165.

Lucking-Reiley, D. (2000): "Auctions on the Internet: What's Being Sold and How?," The Journal of Industrial Economics, 48(3), 227-252.

Matzkin, R. (2003): "Nonparametric Estimation of Nonadditive Random Functions," Econometrica, 71(5), 1339-1375. 
Milgrom, P., and R. Weber (1982): "A Theory of Auctions and Competitive Bidding," Econometrica, 50(5), 1089-1122.

Myerson, R. (1981): "Optimal Auction Design," Mathematics of Operations Research, 6(1), 5873.

Nadaraya, E. (1964): "Some New Estimates for Distribution Functions," Theory of Probability and Its Applications, 15, 497-500.

Neyman, J., And E. L. Scott (1948): "Consistent Estimates Based on Partially Consistent Observations," Econometrica, 16(1), 1-32.

Olley, S., And A. Pakes (1996): "The Dynamics of Productivity in the Telecommunications Equipment Industry," Econometrica, 65(6), 1263-1297.

PAARsch, H. (1997): "Deriving an Estimate of the Optimal Reserve Price: An Application to British Columbia Timber Sales," Journal of Econometrics, 78, 333-357.

PAKes, A. (2008): "Theory and Empirical Work on Imperfectly Competitive Markets," Working Paper.

Pinkse, J., And G. TAn (2005): "The Affiliation Effect in First Price Auctions," Econometrica, $73(1), 263-277$.

Shaked, M., And J. Shanthikumar (1994): Stochastic Orders and their Applications. Academic Press, San Diego, CA.

Silverman, B. (1986): Density Estimation for Statistics and Data Analysis. Chapman Hall, London.

SonG, U. (2004): "Nonparametric Estimation of an eBay Auction Model with an Unknown Number of Bidders," Working Paper.

TAYlor, P. (2007): "NADA Data 2007," Annual report, National Automobile Dealers Association. Yao, S., And C. Mela (2008): "Online Auction Demand," Marketing Science, 27, 861-885. 
Table 1: Auction Summary Statistics

\begin{tabular}{lccccc}
\hline Variable & Mean & Standard Deviation & 25th-tile & Median & 75 th-tile \\
\hline WINNING BID (in \$) & 2948.72 & 2629.63 & 910.00 & 2390.00 & 4290.00 \\
RESERVE PRICE (in \$) & 2691.87 & 2569.76 & 700.00 & 2100.00 & 4000.00 \\
WINNING BID/RESERVE PRICE & 1.25 & 0.58 & 1.03 & 1.10 & 1.25 \\
WINNING BID - RESERVE PRICE (in \$) & 256.84 & 267.45 & 60.00 & 180.00 & 380.00 \\
NUMBER OF UNIQUE BIDS & 4.90 & 2.32 & 3.00 & 4.00 & 6.00 \\
AGE (in months) & 76.47 & 28.40 & 59.00 & 81.00 & 98.00 \\
MILEAGE (in 100,000 km's) & 1.01 & 0.49 & 0.68 & 1.00 & 1.30 \\
AUTOMATIC & 0.58 & 0.49 & 0 & 0 & 1 \\
PASSENGERS & 0.98 & 0.12 & 1 & 1 & 1 \\
MAKE & & & & & \\
HYUNDAI & 0.44 & 0.49 & 0 & 0 & 1 \\
DAEWOO & 0.36 & 0.48 & 0 & 0 & 1 \\
KIA & 0.20 & 0.40 & 0 & 0 & 0 \\
\hline \hline
\end{tabular}

There are 8,589 observations of each variable and the time period is 10/10/2001-11/27/2002.

Table 2: Bidder Summary Statistics

\begin{tabular}{lccccc}
\hline Variable & Mean & Standard Deviation & 25th-tile & Median & 75th-tile \\
\hline DISTANCE (in 100 km's) & 1.04 & 0.80 & 0.42 & 1.06 & 1.06 \\
TOTAL NUMBER OF & 22.30 & 37.23 & 3.00 & 10.00 & 25.00 \\
AUCTIONS WON & & & & & \\
NUMBER OF AUCTIONS & 2.32 & 2.03 & 1.00 & 2.00 & 3.00 \\
$\quad$ WON PER DAY & & & 104.00 & 140.00 & 183.00 \\
$\quad \begin{array}{l}\text { NUMBER OF AUCTIONS } \\
\text { HELD PER DAY }\end{array}$ & 145.58 & 58.04 & & & \\
$\quad$ NUMBER OF DIFFERENT & 9.62 & 10.96 & 2.00 & 5.00 & 13.00 \\
DAYS BIDDING & & & & & \\
\hline
\end{tabular}

The time period is from $10 / 10 / 2001-11 / 27 / 2002$.

All variables are averages across either days or bidders.

The variable DISTANCE measures the distance between the auction house and the centroid of the winning bidder's province. 
Table 3: Winning Bid Determinants

\begin{tabular}{|c|c|c|c|c|c|c|}
\hline & $(1)$ & $(2)$ & $(3)$ & $(4)$ & $(5)$ & $(6)$ \\
\hline$\overline{\mathrm{AGE}}$ & $\begin{array}{c}-0.029^{* * *} \\
(0.0002)\end{array}$ & $\begin{array}{c}-0.029^{* * *} \\
(0.0002)\end{array}$ & $\begin{array}{c}-0.028^{* * *} \\
(0.0002)\end{array}$ & $\begin{array}{c}-0.029^{* * *} \\
(0.0002)\end{array}$ & $\begin{array}{c}-0.028^{* * *} \\
(0.0002)\end{array}$ & $\begin{array}{c}-0.004^{* * *} \\
(0.0002)\end{array}$ \\
\hline MILEAGE & $\begin{array}{c}-0.026^{* *} \\
(0.011)\end{array}$ & $\begin{array}{c}-0.024^{* *} \\
(0.011)\end{array}$ & $\begin{array}{c}-0.030^{* * *} \\
(0.011)\end{array}$ & $\begin{array}{c}-0.024^{* *} \\
(0.011)\end{array}$ & $\begin{array}{c}-0.030^{* * *} \\
(0.011)\end{array}$ & $\begin{array}{c}-0.013^{* *} \\
(0.005)\end{array}$ \\
\hline AUTOMATIC & $\begin{array}{c}0.423^{* * *} \\
\quad(0.01)\end{array}$ & $\begin{array}{c}0.419^{* * *} \\
(0.01)\end{array}$ & $\begin{array}{c}0.411^{* * *} \\
(0.009)\end{array}$ & $\begin{array}{c}0.405^{* * *} \\
(0.01)\end{array}$ & $\begin{array}{c}0.399^{* * *} \\
(0.01)\end{array}$ & $\begin{array}{c}0.091^{* * *} \\
(0.005)\end{array}$ \\
\hline PASSENGERS & $\begin{array}{c}0.391^{* * *} \\
(0.04)\end{array}$ & $\begin{array}{c}0.378^{* * *} \\
(0.04)\end{array}$ & $\begin{array}{c}0.375^{* * *} \\
(0.04)\end{array}$ & $\begin{array}{c}0.374^{* * *} \\
(0.04)\end{array}$ & $\begin{array}{c}0.370^{* * *} \\
(0.04)\end{array}$ & $\begin{array}{c}0.092^{* * *} \\
(0.019)\end{array}$ \\
\hline DISTANCE & $\begin{array}{c}0.032^{* * *} \\
(0.006)\end{array}$ & $\begin{array}{c}0.029^{* * *} \\
(0.006)\end{array}$ & $\begin{array}{c}0.028^{* * *} \\
(0.006)\end{array}$ & $\begin{array}{l}0.012 \\
(0.015)\end{array}$ & $\begin{array}{l}0.013 \\
(0.015)\end{array}$ & $\begin{array}{c}-0.012^{*} \\
(0.007)\end{array}$ \\
\hline LOG RESERVE PRICE & & & & & & $\begin{array}{c}0.749^{* * *} \\
(0.004)\end{array}$ \\
\hline MAKE DUMMIES & YES & YES & YES & YES & YES & YES \\
\hline MODEL DUMMIES & YES & YES & YES & YES & YES & YES \\
\hline MONTH DUMMIES & $\mathrm{NO}$ & YES & YES & YES & YES & YES \\
\hline COLOR DUMMIES & $\mathrm{NO}$ & $\mathrm{NO}$ & YES & $\mathrm{NO}$ & YES & YES \\
\hline PROVINCE DUMMIES & $\mathrm{NO}$ & $\mathrm{NO}$ & $\mathrm{NO}$ & YES & YES & YES \\
\hline $\mathrm{N}$ & 8,589 & 8,589 & 8,589 & 8,589 & 8,589 & 8,589 \\
\hline $\mathrm{R}^{2}$ & 0.836 & 0.840 & 0.846 & 0.841 & 0.847 & 0.966 \\
\hline
\end{tabular}

Dependent variable is the log of the winning bid in each auction.

The data are conditional on the object selling.

${ }^{*},{ }^{* *},{ }^{* *}$ imply significance at the $10 \%, 5 \%$ and $1 \%$ level, respectively. 
Table 4: Reserve Price Determinants

\begin{tabular}{lcccc}
\hline & $(1)$ & $(2)$ & $(3)$ & $(4)$ \\
\hline AGE & $-0.032^{* * *}$ & $-0.032^{* * *}$ & $-0.031^{* * *}$ & $-0.031^{* * *}$ \\
MILEAGE & $(0.0003)$ & $(0.0003)$ & $(0.0003)$ & $(0.0003)$ \\
& -0.019 & -.016 & $-0.026^{* *}$ & $-0.023^{*}$ \\
AUTOMATIC & $(0.013)$ & $(0.013)$ & $(0.013)$ & $(0.013)$ \\
& $0.441^{* * *}$ & $0.434^{* * *}$ & $0.433^{* * *}$ & $0.426^{* * *}$ \\
PASSENGERS & $(0.011)$ & $(0.011)$ & $(0.011)$ & $(0.011)$ \\
& $0.416^{* * *}$ & $0.402^{* * *}$ & $0.395^{* * *}$ & $0.382^{* * *}$ \\
MAKE DUMMIES & $(0.048)$ & $(0.047)$ & $(0.047)$ & $(0.047)$ \\
& YES & YES & YES & YES \\
MODEL DUMMIES & YES & YES & YES & YES \\
MONTH DUMMIES & NO & & & YES \\
COLOR DUMMIES & NO & NO & NO & YES \\
N & & & & YES \\
$\mathrm{R}^{2}$ & 8,589 & 8,589 & 8,589 & 8,589 \\
\hline \hline
\end{tabular}

Dependent variable is the log of the reserve price in each auction.

The data are conditional on the object selling.

${ }^{*},{ }^{* *},{ }^{* * *}$ imply significance at the $10 \%, 5 \%$ and $1 \%$ level, respectively. 


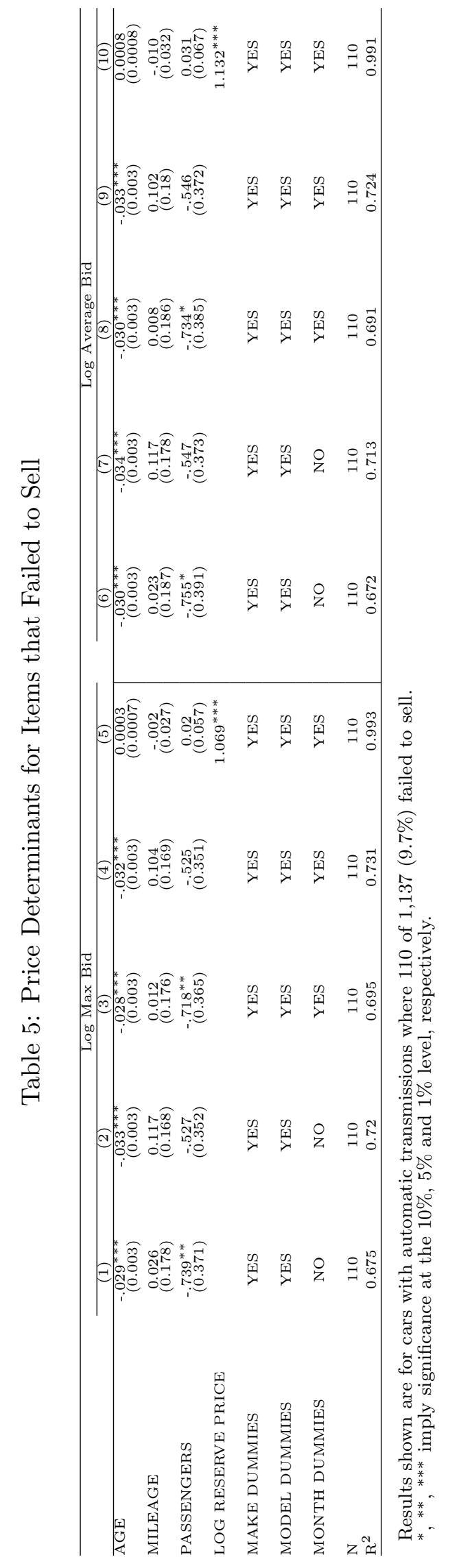


Table 6: Number of Unique Winning Bidders: Averages by Month

\begin{tabular}{lrr}
\hline & \multicolumn{2}{c}{ Year } \\
Month & 2001 & 2002 \\
\hline January & & 67.8 \\
February & & 88.6 \\
March & & 88.0 \\
April & & 79.5 \\
May & & 73.8 \\
June & & 52.0 \\
July & & 55.8 \\
August & & 71.7 \\
September & & 54.3 \\
October & 69.8 & 56.5 \\
November & 59.5 & 40.8 \\
December & 54.5 & \\
\hline
\end{tabular}

Table 7: Percentile Estimates of the Distributions of Bidder Values, at Mileage, Age and Reserve Price Points

\begin{tabular}{lccccc}
\hline & \multicolumn{5}{c}{ Distributions: } \\
Percentile & Fvall & Fv(R1) & Fv(R2) & Fv(R3) & Fv(R4) \\
\hline 1: 5 years, 50,000 kms & & & & & \\
\hline 25 & 7.71 & 7.25 & 7.42 & 7.76 & 8.13 \\
50 & 8.13 & 7.58 & 7.77 & 8.12 & 8.53 \\
75 & 8.48 & 7.90 & 8.13 & 8.43 & 8.90 \\
95 & 8.92 & 8.33 & 8.63 & 8.83 & 9.40 \\
\hline $2: 6.5$ years, 100,000 kms & & & & & \\
\hline 25 & 7.12 & 5.75 & 7.05 & 7.40 & 7.69 \\
50 & 7.71 & 6.24 & 7.48 & 7.75 & 8.06 \\
75 & 8.15 & 6.67 & 7.83 & 8.09 & 8.39 \\
95 & 8.69 & 7.07 & 8.26 & 8.53 & 8.81 \\
\hline $3: 7.5$ years, $125,000 \mathrm{kms}$ & & & & & \\
\hline 25 & 5.70 & 5.42 & 6.99 & 7.16 & 7.42 \\
50 & 7.39 & 5.79 & 7.38 & 7.54 & 7.77 \\
75 & 7.90 & 6.26 & 7.73 & 7.87 & 8.11 \\
95 & 8.44 & 6.88 & 8.18 & 8.31 & 8.55 \\
\hline $4: 8.5$ years, $150,000 \mathrm{kms}$ & \multicolumn{5}{c}{} \\
\hline 25 & 5.09 & 5.18 & 5.45 & 7.00 & 7.25 \\
50 & 6.12 & 5.58 & 5.86 & 7.36 & 7.59 \\
75 & 7.66 & 6.08 & 6.32 & 7.69 & 7.93 \\
95 & 8.21 & 6.71 & 6.88 & 8.14 & 8.37 \\
\hline \hline
\end{tabular}

Results are for 5 passenger Hyundai Sonatas with automatic transmissions sold in month group B. This assumes the number of potential bidders equals 8 . The percentiles cutoffs are in logs. "R\#" signifies a certain reserve price with greater \#'s signifying greater reserve prices. Respectively, they are the 15th, 25th, 50th and 90th percentiles of reserve prices for cars conditional on age and mileage. Fvall ignores unobserved heterogeneity. 
Table 8: Estimates of the Interquartile Range of the Value Distributions

\begin{tabular}{lccccc}
\hline & \multicolumn{5}{c}{ Distributions: } \\
(Mileage, Age) Point & Fvall & Fv(R1) & Fv(R2) & Fv $(\mathrm{R} 3)$ & Fv(R4) \\
\hline$(0.50,5.5)$ & 0.77 & 0.65 & 0.71 & 0.67 & 0.78 \\
$(1.00,6.5)$ & 1.02 & 0.92 & 0.78 & 0.69 & 0.70 \\
$(1.25,7.5)$ & 0.99 & 0.72 & 0.74 & 0.71 & 0.69 \\
$(1.50,8.5)$ & 1.80 & 0.94 & 0.68 & 0.69 & 0.68 \\
\hline \hline
\end{tabular}

Results are for 5 passenger Hyundai Sonatas with automatic transmissions sold in month group B. This assumes the number of potential bidders equals 8 . MILEAGE and AGE are in 100,000 km's and months, respectively. The IQRs are in logs. "R\#" signifies a certain reserve price with greater \#'s signifying greater reserve prices. Respectively, they are the 15th, 25th, 50th and 90th percentiles of reserve prices for cars conditional on age and mileage. Fvall ignores unobserved heterogeneity.

Table 9: Controlling for Within Bid Variation

\begin{tabular}{|c|c|c|c|c|c|c|c|c|c|c|c|c|c|c|c|c|}
\hline \multirow{2}{*}{$\begin{array}{l}\text { Mileage Group } \\
\text { Age Group }\end{array}$} & \multicolumn{4}{|c|}{ (1) } & \multicolumn{4}{|c|}{$(2)$} & \multicolumn{4}{|c|}{ (3) } & \multicolumn{4}{|c|}{ (4) } \\
\hline & $(1)$ & $(2)$ & $(3)$ & (4) & (1) & (2) & (3) & (4) & $(1)$ & $(2)$ & (3) & $(4)$ & (1) & $(2)$ & (3) & (4) \\
\hline Within & 110.8 & 102.2 & 98.1 & 90.3 & 99.1 & 95.5 & 106.0 & 86.0 & 102.7 & 95.2 & 100.6 & 84.3 & 94.2 & 99.6 & 87.3 & 76.4 \\
\hline Across & 2359.0 & 1866.9 & 968.2 & 1644.4 & 2012.6 & 1479.6 & 1607.0 & 1368.5 & 3019.2 & 1574.3 & 1786.3 & 1186.8 & 1872.1 & 1290.0 & 893.9 & 615.1 \\
\hline Across, by Reserve Grou & & & & & & & & & & & & & & & & \\
\hline$(1)$ & 806.9 & 596.6 & 347.9 & 508.8 & 335.0 & 656.8 & 308.0 & 146.4 & 433.9 & 492.6 & 228.1 & 207.9 & 661.9 & 240.1 & 266.5 & 261.0 \\
\hline (2) & 361.7 & 276.3 & 454.6 & 482.9 & 435.8 & 222.4 & 491.2 & 303.9 & 344.9 & 387.5 & 424.9 & 250.8 & 348.5 & 292.0 & 251.7 & 169.4 \\
\hline (3) & 337.5 & 324.8 & 316.0 & 306.8 & 290.8 & 535.4 & 425.8 & 304.5 & 436.9 & 303.6 & 340.2 & 217.8 & 503.1 & 438.3 & 327.6 & 164.4 \\
\hline (4) & 2553.4 & 3116.7 & 978.1 & 1050.7 & 2802.2 & 1743.0 & 1348.7 & 2126.3 & 5183.2 & 1570.1 & 2206.1 & 1570.0 & 2282.5 & 879.0 & 760.7 & 1089.5 \\
\hline
\end{tabular}

Table 10: Estimated Surplus

\begin{tabular}{ccccccc}
\hline Bidders & \multicolumn{2}{c}{$N=3$} & \multicolumn{2}{c}{$N=5$} & \multicolumn{2}{c}{$N=10$} \\
(Mileage, Age) Point & Fvall & Fv(R3) & Fvall & Fv(R3) & Fvall & Fv(R3) \\
\hline 1 & 2006.65 & 1656.48 & 1864.15 & 1475.70 & 1805.47 & 1351.65 \\
2 & 1764.98 & 1266.95 & 1554.70 & 1170.08 & 1445.06 & 1121.64 \\
3 & 1592.91 & 1020.52 & 1350.74 & 927.66 & 1230.16 & 864.67 \\
4 & 1395.57 & 848.15 & 1265.90 & 782.03 & 996.50 & 721.71 \\
\hline
\end{tabular}

This is based on simulating 5000 auctions from each estimated value distribution. 


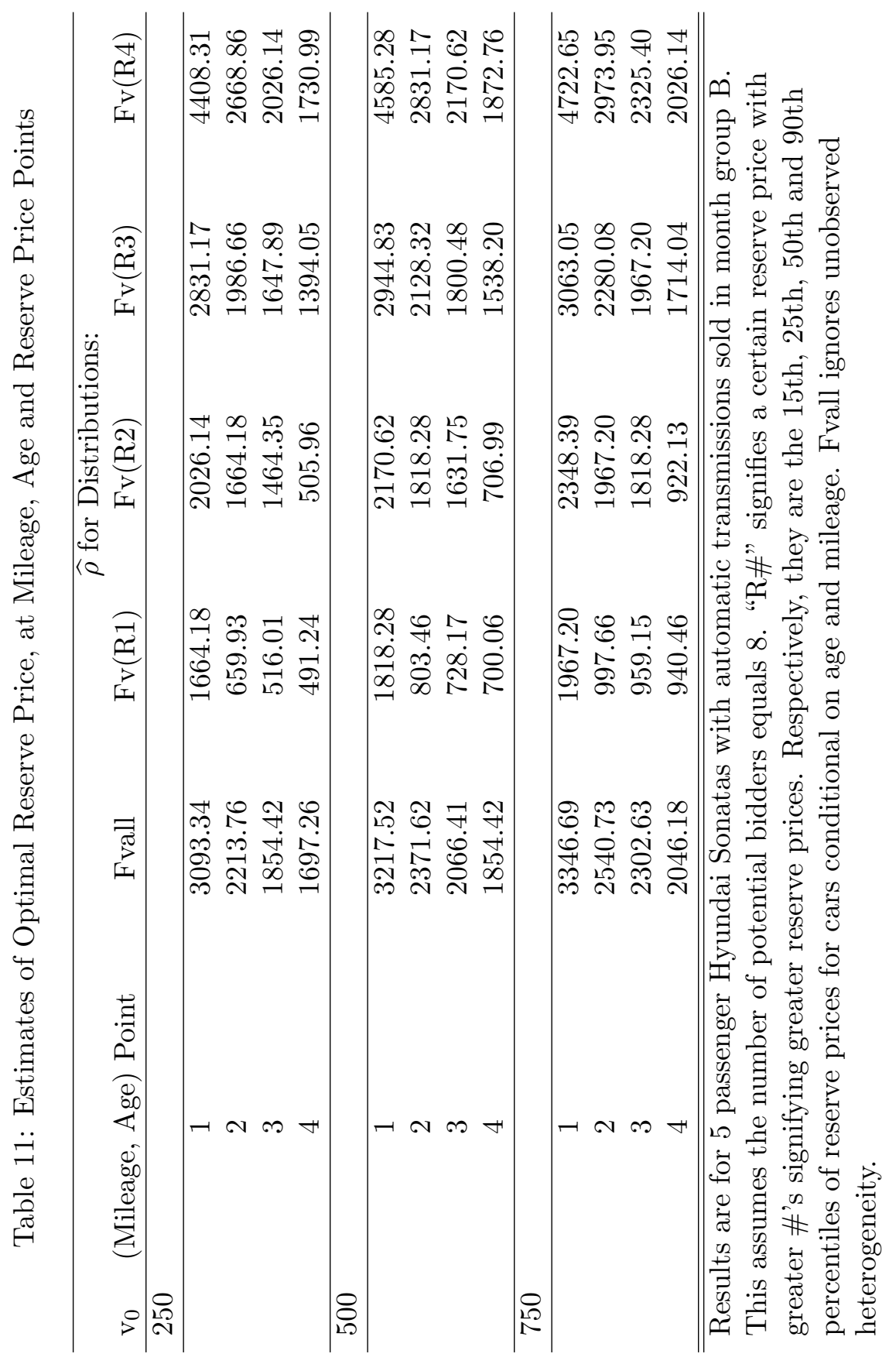


Table 12: Optimal Fixed Fees

\begin{tabular}{ccccccc}
\hline & \multicolumn{2}{c}{ Parameters: } & \multicolumn{4}{c}{ Percentiles of $F^{*}:$} \\
Controlling? & $\gamma$ & $\delta$ & 25 th & 50 th & 75 th & Gains $/$ car \\
\hline No & 0.957 & 0.120 & $\$ 95.39$ & $\$ 191.61$ & $\$ 213.95$ & $\$ 2.87$ \\
Yes & 0.930 & 0.133 & $\$ 89.13$ & $\$ 158.84$ & $\$ 251.78$ & $\$ 10.50$ \\
\hline \hline
\end{tabular}

The table shows the impact of controlling for unobserved heterogeneity when measuring the gains from price discriminating. The gains are measured relative to the current pricing scheme used by the auction house.

Table 13: Monte Carlo Design and Results for Estimating $m$ and $F_{U}$ Nonparametrically

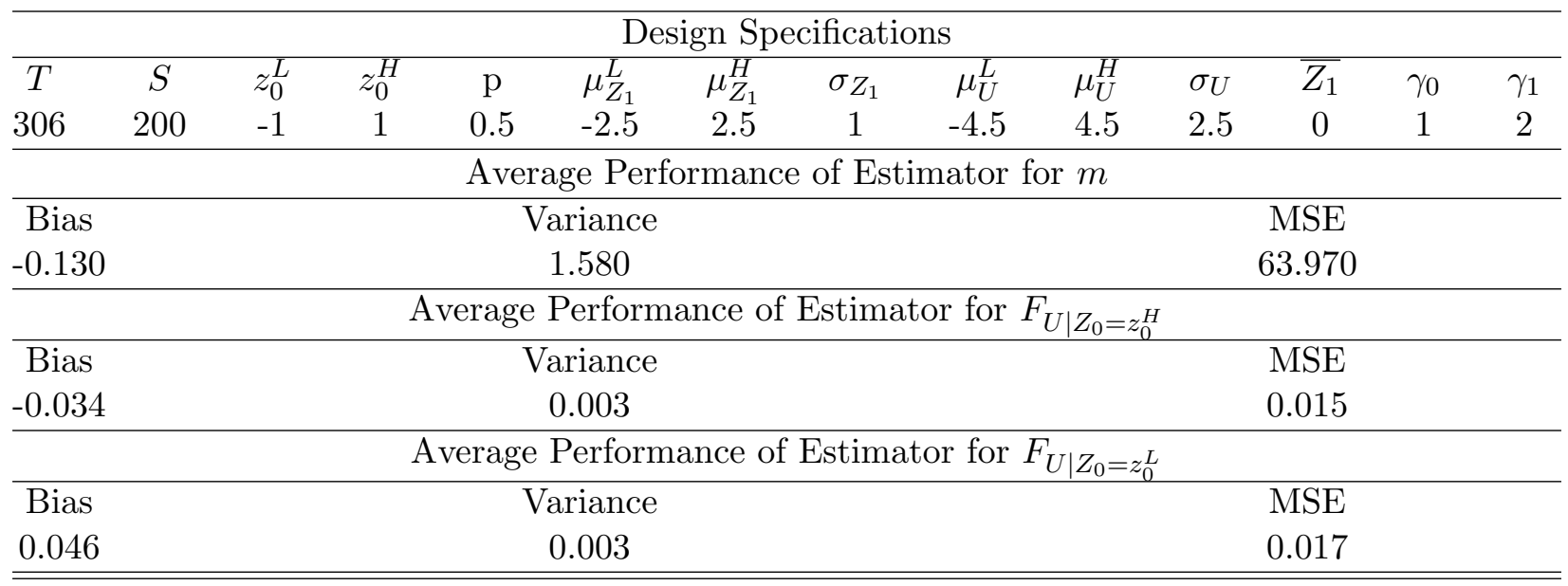

Table 14: Monte Carlo Design for Testing Performance of Estimator of Value Distribution

\begin{tabular}{cccc|ccc}
\hline Parameter & & $\alpha$ & & $\sigma$ & \\
& NI & PI & FI & NI & PI & FI \\
\hline Truth & & 6 & & & 1.5 & \\
Bias & -0.279 & -0.019 & 0.002 & 0.254 & 0.044 & -0.012 \\
Variance & 0.002 & 0.032 & 0.00004 & 0002 & 0.002 & 0.001 \\
MSE & 0.080 & 0.032 & 0.00005 & 0.067 & 0.004 & 0.002 \\
\hline \hline
\end{tabular}

"NI", "PI" and "FI" stand for "No", "Partial" and "Full" Information respectively, the meanings of which are described in the text.

MC Parameters: $M=100, T=500, N_{t}=5$.

$\log L_{N I}=-698.1, \log L_{P I}=-639.2$ and $\log L_{F I}=-614.1$ 

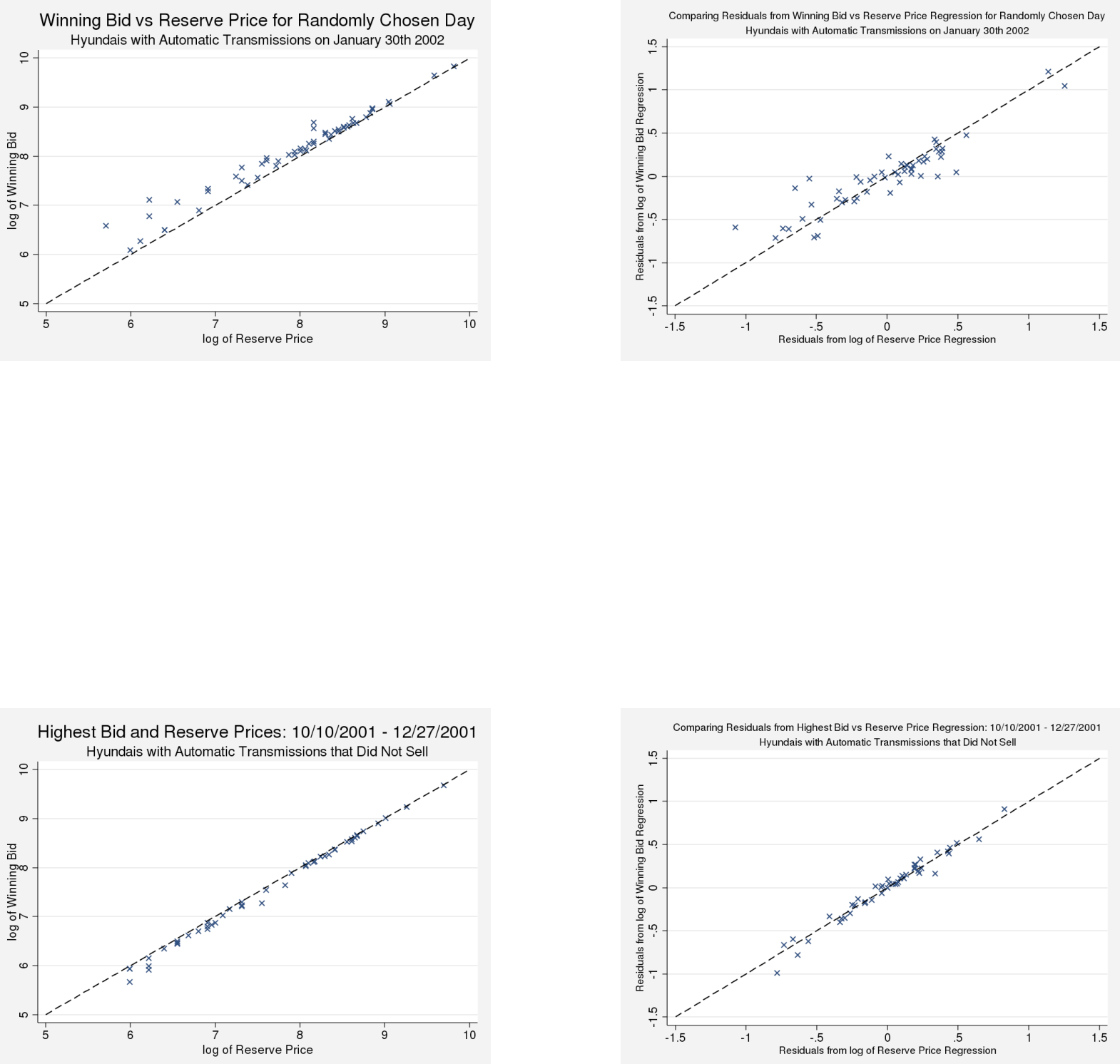

Figure 1: Top row: Plot of winning bid and reserve price and of residuals from regressions of winning bid and reserve price on car characteristics, respectively, for cars that sold on a randomly chosen day. Bottom row: Plot of winning bid and reserve price and of residuals from regressions of winning bid and reserve price on car characteristics, respectively, for cars that did not sell on the same randomly chosen day. 
Figure 2: Estimated Value Distributions, Not Controlling and Controlling for Unobserved Heterogeneity with Confidence Intervals: Automatic, 5 Passenger, Hyundai Sonatas, (MILEAGE,AGE) Point 4, Month Category B

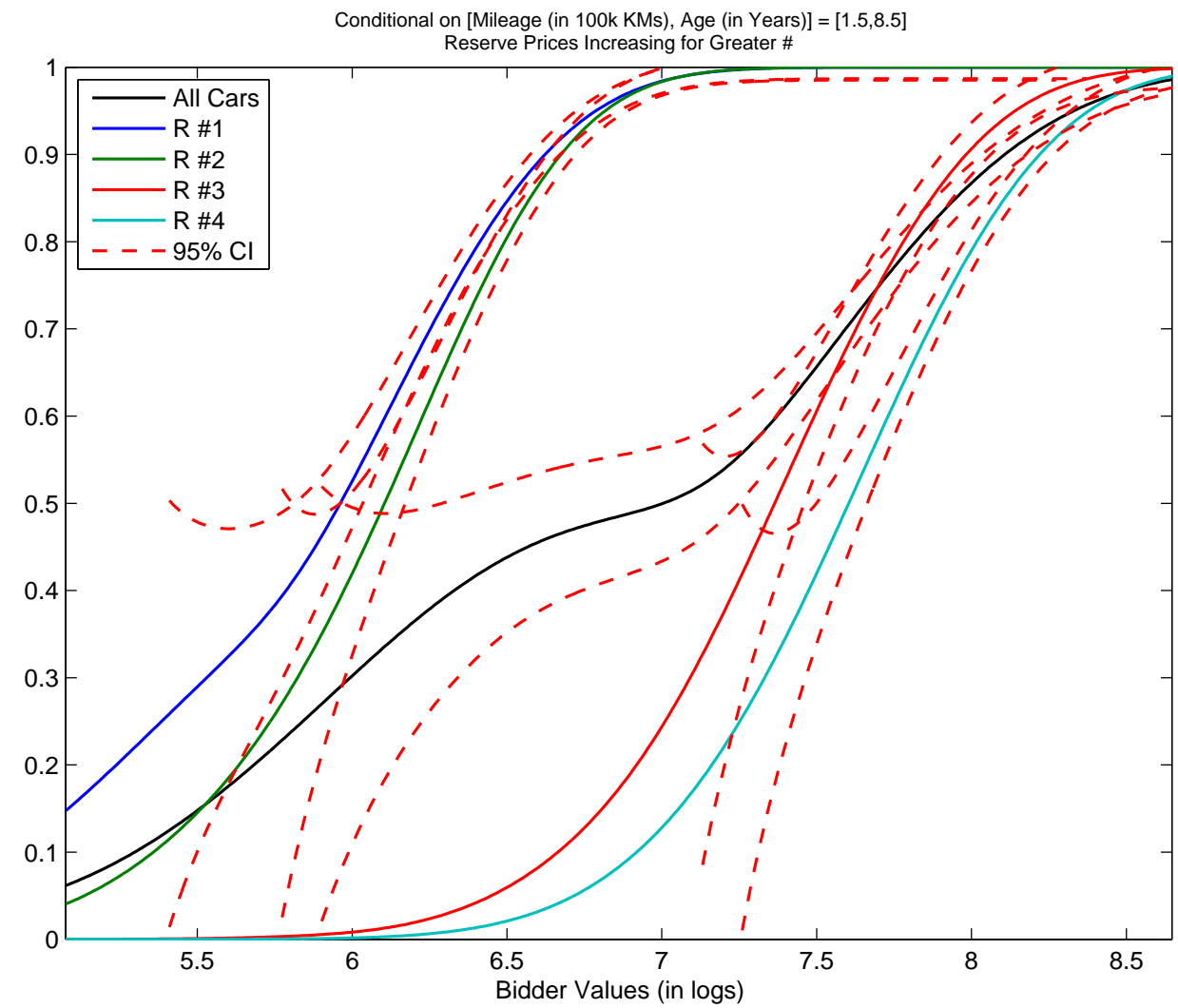


Figure 3: Estimated Value Distributions, Not Controlling and Controlling for Unobserved Heterogeneity: Automatic, 5 Passenger, Hyundai Sonatas, Month Category B
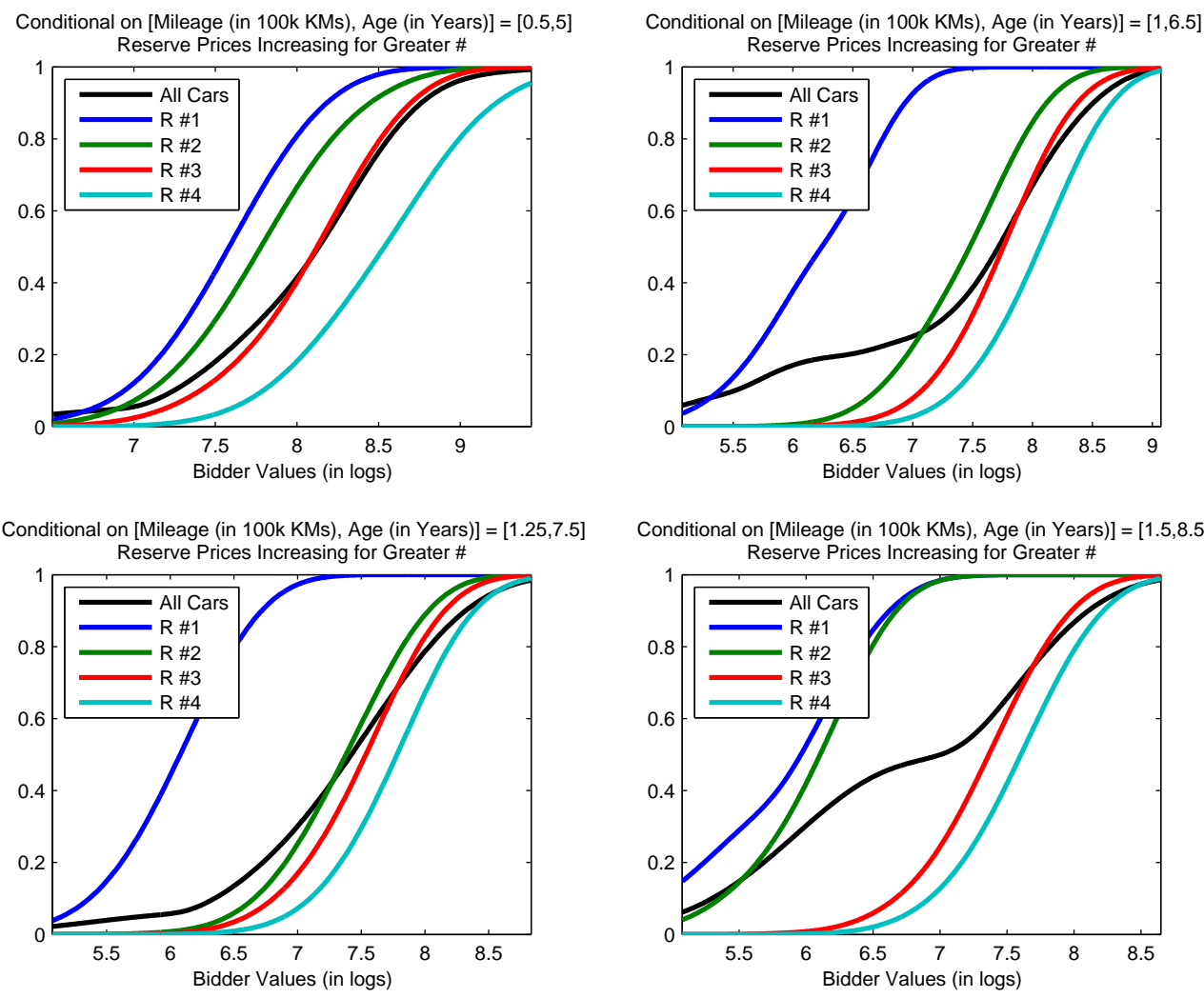
Figure 4: Plot of estimated $5^{\text {th }}$ order polynomial fit of optimal fixed fees per car

Price at Auction House vs. Winning Bid

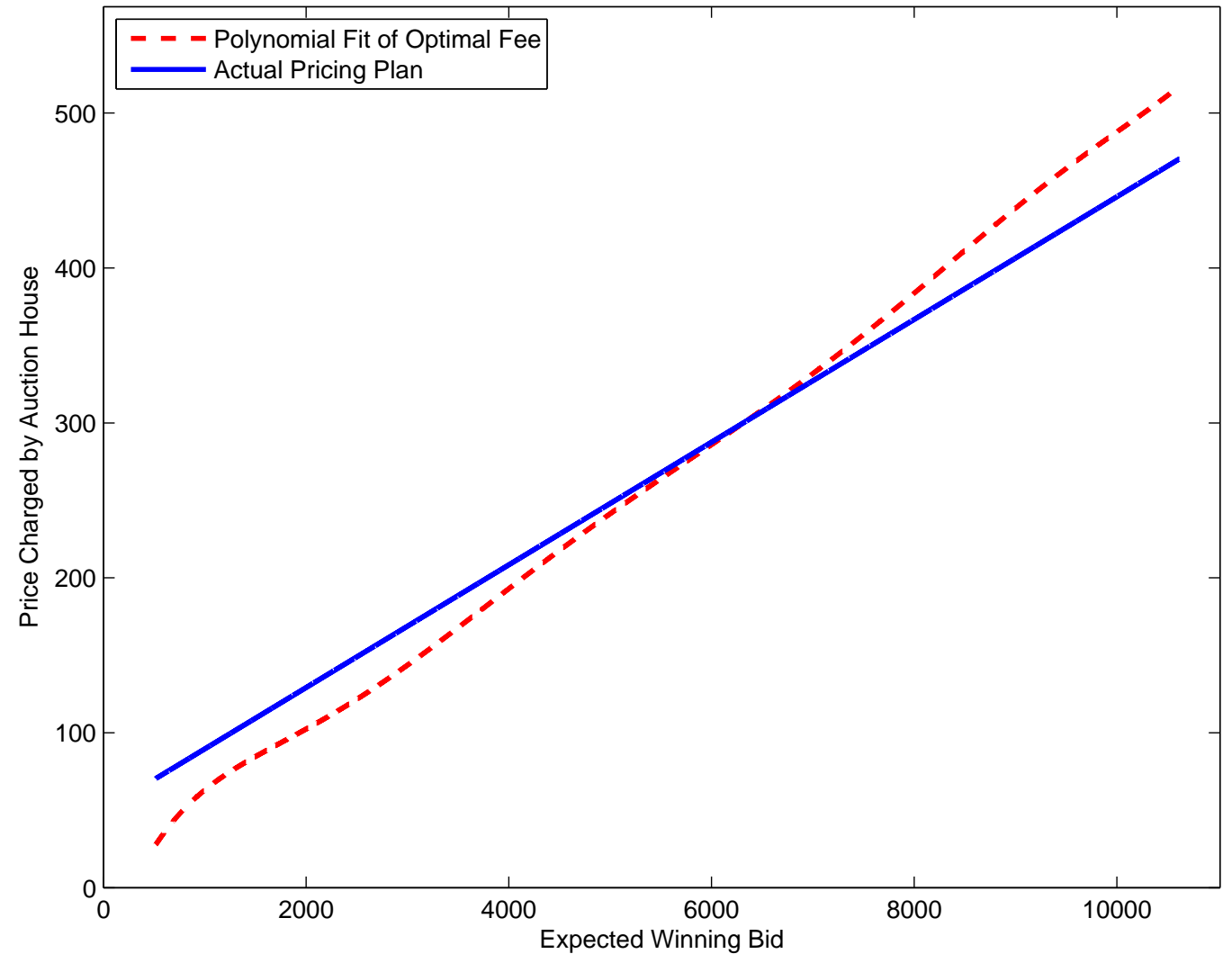


Figure 5: Estimated distribution of unobserved heterogeneity: Monte Carlo results.

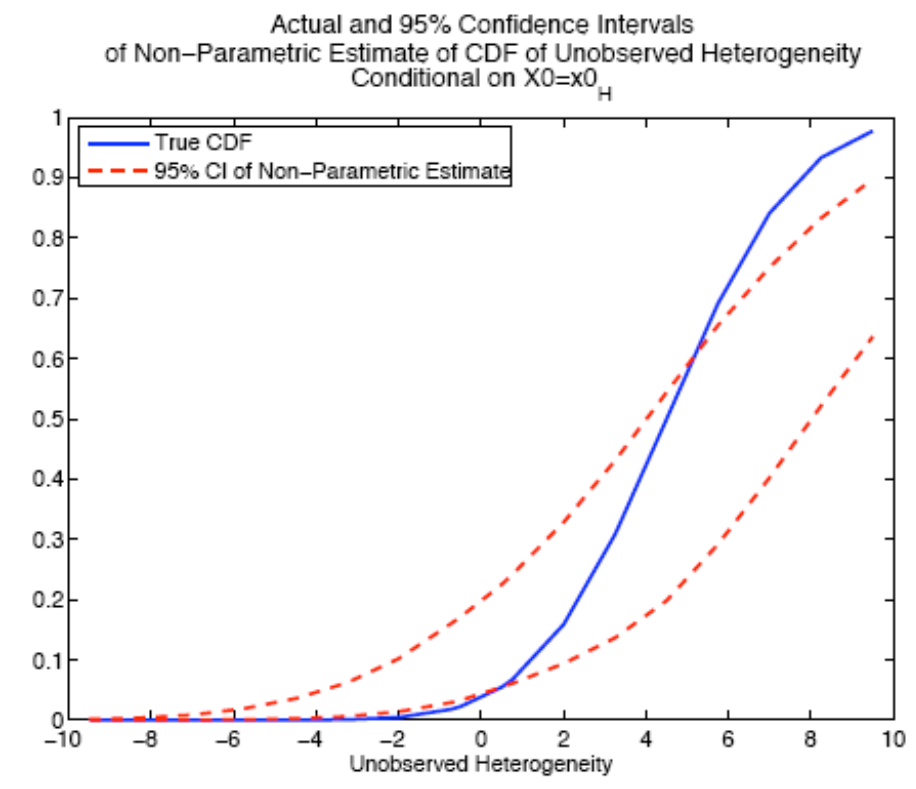

Actual and $95 \%$ Confidence Intervals of Non-Parametric Estimate of CDF of Unobserved Heterogeneity Conditional on $\mathrm{XO}=\mathrm{X} \mathrm{L}_{\mathrm{L}}$

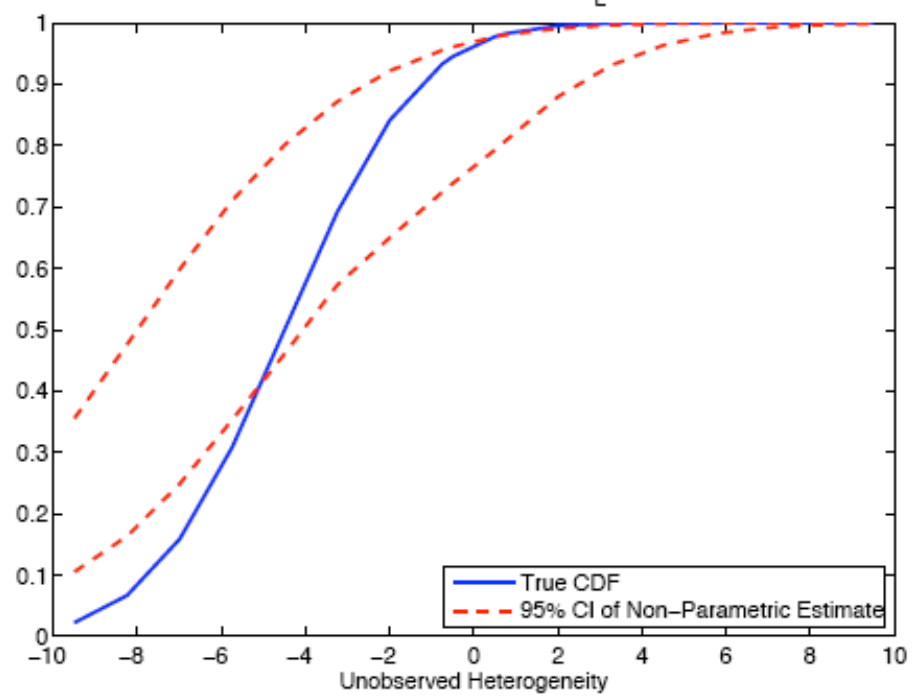


Figure 6: Depiction of optimal reserve price with commission $t$.

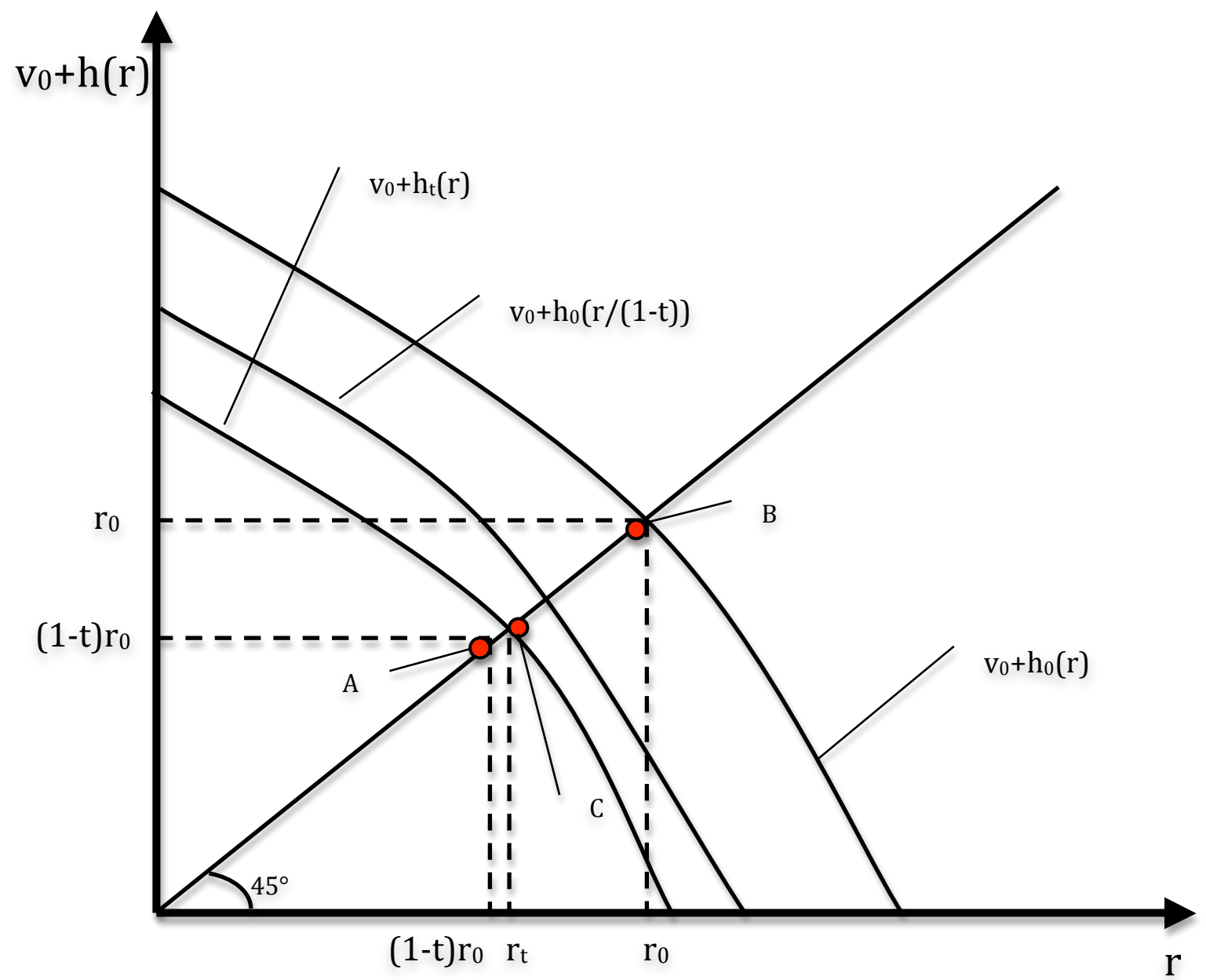

Where:

$$
\begin{aligned}
& h_{0}(r)=\frac{1-F_{v}(r)}{f_{v}(r)} \\
& h_{t}(r)=\frac{1-F_{\tilde{v}}(r)}{f_{\tilde{v}}(r)}
\end{aligned}
$$

